\title{
HATS: Field Observations to Obtain Spatially Filtered Turbulence Fields from Crosswind Arrays of Sonic Anemometers in the Atmospheric Surface Layer*
}

\author{
T. W. Horst, ${ }^{+}$J. Kleissl, ${ }^{\#}$ D. H. Lenschow, ${ }^{+}$C. Meneveau, ${ }^{\#}$ C.-H. Moeng, ${ }^{+}$M. B. Parlange, \\ P. P. Sullivan, + AND J. C. Weil @ \\ ${ }^{+}$National Center for Atmospheric Research, Boulder, Colorado \\ \#The Johns Hopkins University, Baltimore, Maryland \\ ${ }^{\circledR}$ CIRES, University of Colorado, Boulder, Colorado
}

(Manuscript received 3 July 2003, in final form 30 December 2003)

\begin{abstract}
The Horizontal Array Turbulence Study (HATS) field program utilized horizontal, crosswind arrays of sonic anemometers to calculate estimates of spatially filtered and subfilter-scale (SFS) turbulence, corresponding to its partitioning in large-eddy simulations (LESs) of atmospheric flows. Measurements were made over a wide range of atmospheric stability and for $z / \Delta_{f}$ nominally equal to $0.25,0.5,1.0$, and 2.0 , where $z$ is height and $\Delta_{f}$ is the width of the spatial filter. This paper examines the viability of the crosswind array technique by analyzing uncertainties in the filtered turbulence fields. Aliasing in the crosswind direction, caused by the discrete spacing of the sonic anemometers, is found to be minimal except for the spatially filtered vertical velocity and for SFS second moments. In those cases, aliasing errors become significant when the sonic spacing is greater than the wavelength at the peak in the crosswind spectrum of vertical velocity. Aliasing errors are estimated to be of a similar magnitude for the crosswind gradients of filtered variables. Surrogate streamwise filtering is performed by assuming Taylor's hypothesis and using the mean wind speed $U$ to interpret sonic time series as spatial data. The actual turbulence advection velocity $U_{c}$ is estimated from the cross correlation between data from HATS sonics separated in the streamwise direction. These estimates suggest that, for near-neutral stratification, the ratio $U_{c} / U$ depends on the turbulence variable and is typically between 1.0 and 1.2. Analysis of LES turbulence fields for a neutrally stratified boundary layer finds that the correlation between the true spatially filtered SFS stress component $\tau_{13}$ and the same variable obtained with surrogate streamwise filtering exceeds 0.98 for $z / \Delta_{f}$ $>0.25$. Within the limits noted, it is concluded that the horizontal array technique is sufficient for the estimation of resolved and SFS turbulence variables.
\end{abstract}

\section{Introduction}

In three-dimensional numerical simulations of high Reynolds number flows, such as those in the atmospheric boundary layer, limitations of computational resources dictate the choice of a grid spacing that is substantially larger than the smallest scales of turbulence. Large-eddy simulation (LES) addresses this problem by partitioning the turbulent flow field into spatially filtered, resolved-scale eddies and unresolved subfilterscale (SFS) eddies. The fluid dynamics transport equations are spatially filtered in order to explicitly calculate only the resolved-scale eddies, while the effects of subfilter-scale eddies on the resolved-scale flow are parameterized using a so-called SFS model.

\footnotetext{
* The National Center for Atmospheric Research is supported by the National Science Foundation.
}

Corresponding author address: Dr. Tom Horst, National Center for Atmospheric Research, Atmospheric Technology Division, P.O. Box 3000, Boulder, CO 80307-3000.

E-mail: horst@ucar.edu
The common premise of the LES technique is that large eddies, which contain most of the energy and carry most of the fluxes, are properly resolved; while the small or subfilter-scale eddies can be adequately parameterized by assuming that they lie mostly within the inertial range of the turbulence spectrum. Hence, early LES modelers developed SFS models on the basis of assumptions that follow from the structure of finescale turbulence. This appears to be justified in the bulk of the convective boundary layer, where energy-containing eddies are large and well resolved, and thus there is a wide separation between the wavelengths of the energycontaining eddies and those of SFS eddies; there LES solutions have been shown to be insensitive to the SFS treatment (e.g., Nieuwstadt et al. 1993). However, in near-surface or stably stratified regions, energy-containing eddies are small, and the SFS turbulence can become important for the transport of momentum, heat, and other constituents (Mason 1994; Sullivan et al. 2003).

Further refinement of SFS models, to properly simulate these more-demanding turbulence regimes, requires observational data to directly investigate the re- 


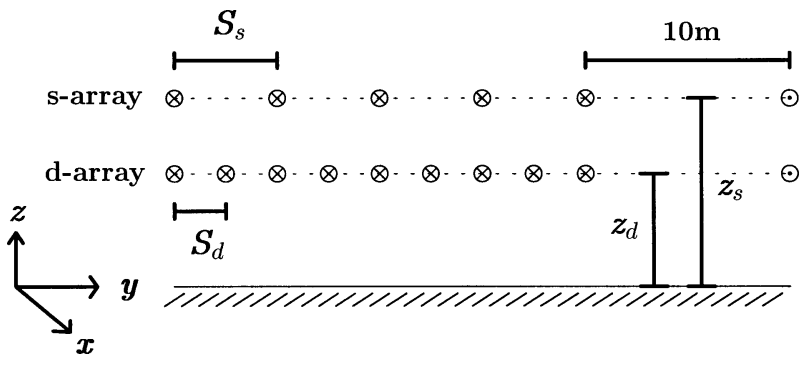

FIG. 1. Generic sonic configuration and the $(x, y, z)$ coordinate system used for data analysis. The sonic anemometers $\otimes$ in the single and double arrays are located at $\left(z_{d}, z_{s}\right)$ above the surface; the crosswind separation between the individual sonics is $\left(S_{s}, S_{d}\right)$. Two reference sonics $\odot$ are used to monitor the possibility of flow interference among the sonics in the $s$ and $d$ arrays.

lationships between resolved and subfilter-scale turbulent fields. Investigations based on analysis of direct numerical simulation (DNS) turbulence fields and on spatially resolved measurements of laboratory flows have been limited to low Reynolds numbers and neutral stratification (Meneveau and Katz 2000). Atmospheric surface-layer turbulence does not have these limitations, but most existing atmospheric data are inadequate because traditional field experiments measure a time series of turbulent motions at a single point, while LES entails spatially filtered turbulence variables.

A method for measuring both spatially filtered and SFS motions in the atmosphere was recently proposed by Tong et al. (1998, hereafter TWKB). This innovative technique uses two horizontal lines of three-component sonic anemometer-thermometers which are oriented transverse to the prevailing wind direction as shown in Fig. 1. Using a crosswind filter composed of five sonics, a primary line of nine sonic anemometers provides spatially filtered and SFS fields of velocity and virtual temperature at the locations of the central five anemometers, and thus can also provide double-filtered flow variables and crosswind gradients of resolved-scale variables at the central sonic. Taylor's hypothesis is assumed in order to use time series data to estimate spatial averages and gradients in the streamwise direction. A second line of five sonic anemometers, displaced vertically from the primary line, is used to calculate vertical gradients of the resolved-scale fields. This general technique has been implemented in field measurement projects by Tong et al. (1999), Porté-Agel et al. (2000b, 2001), and most recently in the Horizontal Array Turbulence Study (HATS) field project executed in September 2000 near Kettleman City, California. A major goal of HATS was to extend significantly the ranges of atmospheric stability and the ratio of height to filter scale over those obtained in the previous, pioneering field studies.

In this paper we describe the design and implementation of the HATS field project (section 2) and examine in detail the accuracy of the horizontal array technique for estimation of resolved-scale and SFS turbulence quantities. TWKB investigated the accuracy of this tech- nique by using turbulence fields generated in an LES of a moderately convective boundary layer to compare array- and time-filtered variables to those obtained by direct 2D spatial filtering. In section 3 we use the HATS field data to investigate the accuracy of the discrete approximation of the crosswind filter over wide ranges of atmospheric stability, filter width, and sonic spacing. Our analysis uses spatially local filters in both horizontal directions, in contrast to TWKB's combination of a spectral-cutoff filter in the streamwise direction and a discrete approximation to a cutoff filter in the crosswind direction. In section 4 we examine the use of Taylor's hypothesis for streamwise filtering, first determining the turbulence advection velocity with HATS field data and then using a neutrally stratified LES simulation to extend the TWKB analysis to a wide range of the ratio of height to filter width. Finally, in section 5, we investigate the accuracy of the finite-difference estimates of spatial gradients of resolved-scale velocities and temperature. This last topic was not investigated by TWKB, but is crucial to examination and development of SFS parameterizations.

\section{HATS field observations}

\section{a. Measurement design}

The HATS field measurement design is based on the transverse array technique proposed by TWKB. The sonic configuration consists of a primary horizontal line of nine equally spaced sonic anemometers mounted at one height and a parallel line of five sonic anemometers at a second height (Fig. 1). The two lines of sonics are oriented perpendicular to the prevailing wind direction so that crosswind-filtered data can be estimated by calculating a weighted sum of the sonic anemometer data at each height, for example,

$$
\mathbf{u}^{a}(\mathbf{x}, t)=\sum_{i=-2}^{2} C_{i}^{a} \mathbf{u}\left(x_{1}, x_{2}+i S, x_{3}, t+i \delta t\right),
$$

where $\mathbf{u}$ is the unfiltered velocity $\left(u_{1}, u_{2}, u_{3}\right), \mathbf{x}=$ $\left(x_{1}, x_{2}, x_{3}\right), \mathbf{u}^{a}$ is the crosswind or array-filtered velocity, and $S$ is the spacing of the sonic anemometers in the $x_{2}$ direction. The crosswind filter encompasses five adjacent sonics and the filter shape depends on the weighting coefficients $C_{i}^{a}$. In reality, the wind direction will seldom be exactly normal to the transverse array. Therefore, assuming Taylor's hypothesis, the observed data are projected onto a line normal to the wind direction by applying time lags $i \delta t$ to the data from each sonic, where $\delta t=S \sin \Theta / U_{c}$. Here $\Theta$ is the mean wind direction relative to the array normal, averaged over all sonics at that height, and $U_{c}$ is the eddy advection velocity, the speed at which turbulence is advected in the streamwise direction. This reduces the effective crosswind spacing of the anemometers, and thus also the filter width, by the factor $\cos \Theta$.

Streamwise filtering is approximated by invoking 
TABLE 1. HATS transverse array dimensions. PDT is Pacific daylight time.

\begin{tabular}{lcccrr}
\hline \hline $\begin{array}{c}\text { Start } \\
(\mathrm{PDT})\end{array}$ & $\begin{array}{c}\text { End } \\
(\mathrm{PDT})\end{array}$ & $\begin{array}{c}z_{d} / \Delta_{f} \\
\left(\Delta_{f}=4 S_{d}\right)\end{array}$ & $\begin{array}{c}z_{d} \\
(\mathrm{~m} \mathrm{AGL})\end{array}$ & $\begin{array}{c}S_{d} \\
(\mathrm{~m})\end{array}$ & $\begin{array}{c}S_{s} \\
(\mathrm{~m})\end{array}$ \\
\hline 1700 31 Aug & 08008 Sep & 0.26 & 3.45 & 3.35 & 6.90 \\
1100 9 Sep & $122515 \mathrm{Sep}$ & 0.50 & 4.33 & 2.17 & 8.66 \\
1725 15 Sep & $130024 \mathrm{Sep}$ & 1.00 & 8.66 & 2.17 & 4.33 \\
1730 25 Sep & 07001 Oct & 2.08 & 4.15 & 0.50 & 5.15 \\
\hline
\end{tabular}

Taylor's hypothesis and calculating a similar weighted sum of the time series data at each measurement location. Thus two-dimensional horizontal filtering is estimated by calculating a weighted sum of the crosswindfiltered data,

$$
\tilde{\mathbf{u}}(\mathbf{x}, t)=\sum_{j=-n}^{n} C_{j}^{t} \mathbf{u}^{a}\left(\mathbf{x}, t+j t_{s}\right),
$$

where $\tilde{\mathbf{u}}$ is the resolved-scale velocity, $t_{s}$ is the time interval between sonic data samples, $C_{j}^{t}$ are the weighting coefficients of the streamwise filter, and the width of the streamwise filter is $2 n U_{c} t_{s}$, which for isotropic filtering in $x_{1}$ and $x_{2}$ should be equivalent to the width of the crosswind filter. Finally, the subfilter-scale velocities are

$$
\mathbf{u}^{\prime}(\mathbf{x}, t)=\mathbf{u}(\mathbf{x}, t)-\tilde{\mathbf{u}}(\mathbf{x}, t) .
$$

Identical two-dimensional filtering is applied to the measured turbulent temperature field, $\theta$.

Formally, LES models filter the equations in three dimensions, and thus the resolved and SFS fields determined from our observations should also be filtered in all three dimensions. However, TWKB conclude on the basis of their analysis and the results of other investigators that two-dimensional, horizontal filtering can provide adequate data for testing SFS models and studying SFS dynamics. They argue that "the variance in the high-wavenumber vertical modes of the calculated resolvable-scale velocity is small compared to that of the total SFS modes." TWKB support their argument by computing two- and three-dimensionally filtered vertical velocities from high-resolution LES simulations of the atmospheric boundary layer and showing that the two are "essentially indistinguishable."

Subfilter-scale second moments are defined as, for example, $\tau_{k l} \equiv \widetilde{u_{k} u_{l}}-\tilde{u}_{k} \tilde{u}_{l}$, and the spatially filtered second moments $\widetilde{u}_{k} u_{l}$ are calculated by applying the filtering operation of Eqs. (1) and (2) to the product $u_{k} u_{l}$. However, it is also of interest to use Eq. (3) to decompose SFS second moments as, for example,

$$
\widetilde{u_{k} u_{l}}=\widetilde{\tilde{u}_{k} \tilde{u}_{l}}+\widetilde{\tilde{u}_{k} u_{l}^{\prime}}+\widetilde{u_{k}^{\prime} \tilde{u}_{l}}+\widetilde{u_{k}^{\prime} u_{l}^{\prime}}
$$

(e.g., Sullivan et al. 2003). In order to measure these four components, $\tilde{u}_{k}, \tilde{u}_{l}, u_{k}^{\prime}$, and $u_{l}^{\prime}$ must be known at five adjacent $x_{2}$ locations, so that a second five-point filtering operation can be performed on the products. Thus, nine equally spaced sonic anemometers are needed, such that five overlapping five-sonic subarrays can be formed to calculate the double-filtered components of the SFS stress at the center point, for example, $\widetilde{\tilde{u}_{k} u_{l}^{\prime}}$. Although the original purpose of the nine-sonic array was double filtering, it will be shown in section $3 \mathrm{~b}$ that data from this array also enables evaluation of the performance of the crosswind filter.

In order to investigate the performance of eddy-viscosity-based SFS models and the production of SFS energy by the resolved scales, it is additionally necessary to measure the resolved-scale velocity and temperature gradients in all three directions. The ability to calculate resolved-scale variables at five adjacent locations in the primary line of nine sonics enables computation of finite-difference estimates of the crosswind gradients of those quantities. Similarly, Taylor's hypothesis can be used to estimate streamwise gradients from the time series data. Finally, a separate line of five sonics is used to spatially filter velocity and temperature at a second height, which permits estimation of vertical gradients of resolved-scale quantities. The second line of sonics is parallel to and either above or below the primary line of sonics, so that the central sonics of both lines have the same horizontal coordinates, for example, Fig. 1.

The goal of the HATS field project was to investigate the turbulent interaction between resolved and subfilter scales over wide ranges of atmospheric stability and filter size. The nature of the interaction is expected to differ depending on whether the filter cutoff wavelength is in the energy-containing range or in the inertial range of the turbulence spectrum. That is, the physics of the interaction will depend on the ratio of the filter width $\Delta_{f}$ to the scale of the energy-containing eddies. The latter scale is a function of both height $z$ and stability $z / L$, where $L$ is the Obukhov length (e.g., Kaimal and Finnigan 1994), and therefore the ratio $z / \Delta_{f}$ was used as a design parameter for the geometry of the sonic configurations. A similar design philosophy was used in the measurements reported by Porté-Agel et al. (2001).

Based on current LES capabilities and practice, practical logistical considerations, and the recent results of Porté-Agel et al. (2001), we chose to investigate four values of $z / \Delta_{f}: 0.25,0.5,1$, and 2 , where $\Delta_{f}=4 S$. The actual values of sonic spacings and heights used in the field are listed in Table 1, along with the start and end times of data collection for each of the four configurations. Here the primary line of nine sonics is denoted 
by the subscript $d$ (referring to the double filter) and the secondary line of five sonics is denoted by the subscript $s$ (single filter). In order to investigate a wide range of atmospheric stability, data were collected continuously from each of the four configuration for 6-9 days.

In order to maximize the measured differences used to estimate the vertical gradients of resolved-scale wind and temperature, while making the spatial resolution of the vertical gradient estimates comparable to that of the horizontal gradient estimates, the ratio of the heights of the two lines of sonics was chosen to be a factor of 2 for all configurations except the last. Horizontal gradients are calculated using a fourth-order central-difference scheme that utilizes resolved-scale quantities with spacings of $\pm S_{d}$ and $\pm 2 S_{d}$. It is shown in section 5 that this fourth-order central-difference scheme has a spatial resolution intermediate between those for simple two-point finite differences with spacings of $S_{d}$ and $2 S_{d}$. Thus the ratio of the spatial resolutions of the vertical and horizontal gradient estimates, roughly $\left|z_{d}-z_{s}\right| /$ $1.5 S_{d}$, is 0.7 for the first configuration and 1.3 for the other three. Finally, the spacing of the sonics in the single-filter array of sonics, $S_{s}$, was chosen to achieve the same value of $z / \Delta_{f}($ or $z / S)$ at both heights.

Four supplementary, instrumented towers were located approximately $60 \mathrm{~m}$ crosswind from the sonic filter arrays. Investigations of Taylor's hypothesis have found that the eddy advection speed $U_{c}$ generally exceeds the mean wind speed by factors as large as 1.2 (e.g., Powell and Elderkin 1974). In order to directly measure the eddy advection speed, two towers were erected with a relative displacement parallel to the prevailing wind direction, and each was instrumented with two sonics at the same heights as the sonic filter arrays. The separation of these two towers was $26.8 \mathrm{~m}$ for the first configuration and $17.3 \mathrm{~m}$ for the other three. Except for the fourth configuration, this corresponds to the span of the nine-sonic $d$ array. These data are discussed in section $4 \mathrm{a}$. In order to document the micrometeorological environment of the detailed turbulence measurements, two additional towers were used to measure profiles of temperature, humidity, and wind speed and direction at five levels between 1 and $10 \mathrm{~m}$.

\section{b. Kettleman City field site}

The site for the HATS field study was selected to satisfy several criteria. In order to simplify interpretation of the observations, this study required a reasonably homogeneous site, particularly one with a spatially uniform surface roughness. For the same reason, it was desired that the turbulence measurements be made above the roughness sublayer (conservatively 100 times the roughness height $z_{o}$ ), requiring a reasonably smooth site with a roughness height on the order of $3 \mathrm{~cm}$ or less. Since one of the goals of the field study was to make measurements with a wide range of thermal stratification, including quite stable conditions at night, a site was sought with weather conditions that featured predominantly clear skies. Most importantly, aligning the sonic filter arrays transverse to the wind direction required that the wind direction be highly predictable and not vary significantly between day and night. These conditions were found to be satisfied in the San Joaquin Valley of southern California. During the summer months, the valley has mostly clear skies and the wind direction is very persistent, both day and night, from the north to northwest. Large expanses of the valley feature naturally flat topography and, in addition, agricultural fields have been accurately leveled to enable flood irrigation.

The HATS measurement site was located about 3.5 miles $(5.6 \mathrm{~km})$ ENE of Kettleman City, California, at the southeast corner of a 3-mile-square area of unplanted farmland. The surface was covered with various mixtures of crop stubble and weeds, and the fields were crossed at roughly $60-\mathrm{m}$ intervals by irrigation check dams that were oriented east to west and rose about 20$25 \mathrm{~cm}$ above the level of the field. An aerodynamic displacement height $d_{0}=32 \mathrm{~cm}$ and a surface roughness length of $2 \mathrm{~cm}$ were calculated from near-neutral wind profiles at the site.

\section{c. Data collection}

The HATS field measurements were obtained from the Integrated Surface Flux Facility (ISFF), which is maintained, deployed, and operated by the Atmospheric Technology Division of the National Center for Atmospheric Research. As indicated in Table 1, data collection extended from 31 August to 1 October 2000. Sixteen identical Campbell Scientific three-component sonic anemometer-thermometers (CSAT3) were used for the two horizontal lines of sonics that composed the spatial-filter arrays, as well as for two sonic anemometers on a nearby reference tower that were used to monitor the possibility that distortion of the flow by one sonic might contaminate the measurements of adjacent sonics (Fig. 1). The CSAT3 sonic anemometers were programmed to output data in the "single measurement" mode at a rate of $20 \mathrm{~s}^{-1}$. A detailed discussion of HATS sonic anemometer data processing and quality control analysis can be found online at www.atd.ucar.edu.

\section{Discrete spatial filters}

\section{a. Filter response functions}

We begin our investigation of the performance of the HATS discrete spatial filters with an examination of the filter response functions. The spatial filtering operation is defined by the general formula

$$
\tilde{\mathbf{u}}(\mathbf{x}, t)=\int G(\mathbf{r}) \mathbf{u}(\mathbf{x}-\mathbf{r}, t) d \mathbf{r},
$$

where the filter function $G$ is assumed to be homoge- 
neous, that is independent of $\mathbf{x}$. For the horizontal array technique, $\mathbf{r}=\left(r_{1}, r_{2}\right)$ and $G=G_{1}\left(r_{1}\right) G_{2}\left(r_{2}\right)$. Equation (5) is evaluated with the field data using the numerical integration (also called quadrature) expressed in Eqs. (1)-(2). Two filter functions have been used to analyze the HATS data: a top-hat or box filter in the crosswind direction and a Gaussian filter in the streamwise direction. A simple top-hat filter is used in the crosswind direction because of the small number of data points available for evaluation of Eq. (1). The top-hat filter is implemented with the data from $n$ sonic anemometers with equal separation $S$, using trapezoidal weighting, $C_{i}^{a}=(0.5,1, \ldots, 1,0.5) /(n-1)$. The effective width of the filter is $\Delta_{f}=(n-1) S$.

The performance of the spatial filters is revealed most clearly in wavenumber space. By the convolution theorem, the Fourier transform of the filtered velocity is equal to the product of the Fourier transform of the unfiltered velocity and the Fourier transform of the filter function, that is,

$$
\hat{\tilde{\mathbf{u}}}(\mathbf{k})=\hat{G}(\mathbf{k}) \hat{\mathbf{u}}(\mathbf{k}),
$$

where $\mathbf{k}=\left(k_{1}, k_{2}\right)$ is the wavenumber and $\hat{a}$ denotes the Fourier transform of $a$. The Fourier transform of the filter function, $\hat{G}$, is here referred to as the filter response function. For a discrete filter with $2 n+1$ equally spaced data points separated by a distance $\mathbf{S}$, for example, five sonic locations, the response function for a symmetrical set of filter weights $\left(C_{j}=C_{-j}\right)$, is following TWKB,

$$
\hat{G}(\mathbf{k})=\sum_{j=-n}^{n} C_{j} \cos (j \mathbf{S} \cdot \mathbf{k}) .
$$

For a continuous, one-dimensional top-hat filter, the response function is

$$
\hat{G}(k)=\sin \left(\Delta_{f} k / 2\right) /\left(\Delta_{f} k / 2\right),
$$

(e.g., Pope 2000, p. 563) where $k$ is the wavenumber component parallel to the direction in which the spatial filtering is applied.

Figure 2 shows the response functions for discrete five-point and continuous top-hat filters of width $4 S$, plotted as a function of normalized wavenumber $k S$. The response function of the discrete top-hat filter is shown only for $k \leq k_{N}=\pi / S$, the Nyquist wavenumber of the filter. Since the discrete filter cannot resolve turbulent fluctuations with wavelengths smaller than $2 S$, energy at wavenumbers greater than $\pi / S$ is aliased to smaller wavenumbers. The response function of the five-point top-hat filter matches the equivalent continuous filter fairly well. It drops from unity at low wavenumbers to the first zero at $k S=\pi / 2$ a little more slowly than the continuous filter, and it noticeably undershoots the continuous filter at its minimum. Note that the response function of the discrete filter equals zero at the Nyquist wavenumber, eliminating aliasing at that point.

In the streamwise direction, assumption of Taylor's hypothesis permits using the measured time series as a

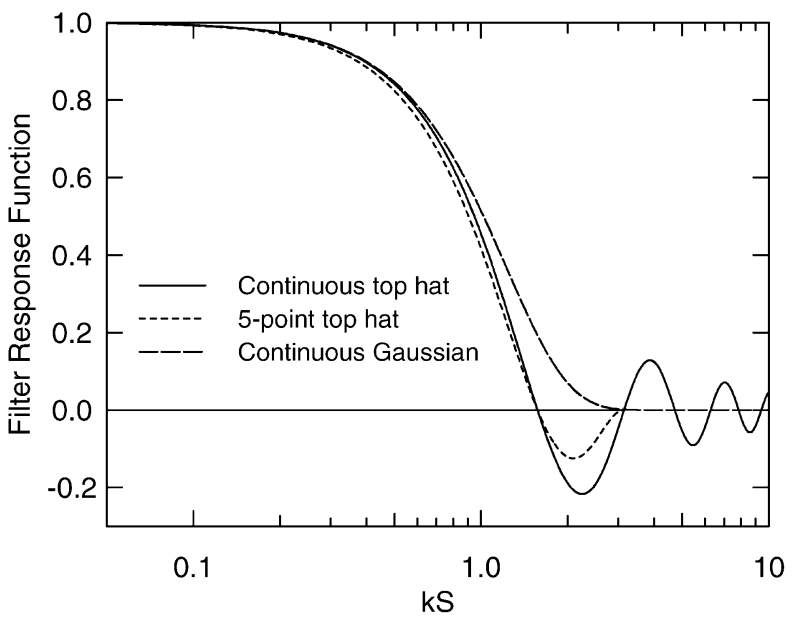

FIG. 2. Response functions of discrete filters used for 2D spatial filtering. Here, $\Delta_{f}=4 S$ for the crosswind top-hat filters, and $\sigma=$ $2 S / \sqrt{3}$ for the streamwise Gaussian filter.

surrogate for spatial sampling. The sonic data were sampled at $20 \mathrm{~s}^{-1}$ and hence the surrogate spatial separation of the data points, $U_{c} t_{s} \approx 15 \mathrm{~cm}$, is generally much smaller than the crosswind sonic spacing. As a consequence, the discrete streamwise filter is composed of significantly more data points than the crosswind filter, and a filter function can be used that has less ringing at high wavenumbers than the top-hat filter. We choose to follow Leonard (1974) and use a Gaussian filter with a second moment equal to that of a continuous top-hat filter of width $\Delta_{f}$,

$$
\begin{aligned}
& G(r)=\left(\frac{6}{\pi \Delta_{f}^{2}}\right)^{1 / 2} \exp \left(-\frac{6 r^{2}}{\Delta_{f}^{2}}\right), \\
& \hat{G}(k)=\exp \left(\frac{-k^{2} \Delta_{f}^{2}}{24}\right) .
\end{aligned}
$$

Because the number of data points in the streamwise filter depends on $U_{c}$ and is, in most cases, considerably greater than for the crosswind filter, we present the continuous Gaussian filter in Eqs. (9) and (10) and also show the response function of the continuous filter in Fig. 2.

\section{b. Discrete crosswind filter performance}

If the power spectra of velocity and temperature do not decrease sufficiently rapidly above the Nyquist wavenumber of a discrete spatial filter, aliasing will materially contaminate the filtered data by removing turbulence from the subfilter scales and translating it into the resolved scales. By design, the half-power points for the crosswind and streamwise filters are almost identical: $k_{1 / 2}=0.70 / S$ for the top-hat filter and $k_{1 / 2}=0.72 /$ $S$ for the Gaussian filter, where $\hat{G}\left(k_{1 / 2}\right) \equiv 1 / \sqrt{2}$. However, the Nyquist wavenumber for the crosswind filter, $k_{N}=\pi / S$, is much smaller than that for the streamwise 
filter, $k_{N}=\pi / U_{c} t_{s}$. Thus aliasing in the crosswind direction is potentially much greater than in the streamwise direction.

Because of the small number of sonics in the horizontal arrays, the crosswind resolution of the HATS data is not sufficient for a satisfactory direct analysis of the aliasing errors. As an alternative, the performance of the discrete crosswind filter is investigated by applying the filter in the streamwise direction where the data have higher spatial resolution $U_{c} t_{s}$. The crosswind filter performance is evaluated using trapezoidal, top-hat quadratures in both the streamwise and crosswind directions. In the crosswind direction, the maximum possible resolution is achieved by using all nine sonics of the $d$ arrays to form a filter of width $8 S \cos \Theta$. In the streamwise direction, we take advantage of the high temporal, and hence spatial, resolution to apply both low- and high-resolution quadratures to the data. For the lowresolution quadratures, the time series data are subsampled at intervals $\delta x=m S \cos \Theta$ and spatially averaged with top-hat filters of width $8 S \cos \Theta$ matching that in the crosswind direction. Thus the streamwise filter, Eq. (2), becomes

$$
\tilde{\mathbf{u}}(\mathbf{x}, t)=\sum_{j=-n}^{n} C_{j}^{t} \mathbf{u}^{a}\left(\mathbf{x}, t+j \delta x / U_{c}\right),
$$

with $n=4 S \cos \Theta / \delta x=4 / m$. Equation (11) is applied with sample spacings $\delta x$ specified by $m=4,2$, and 1 , corresponding to three-, five-, and nine-point quadratures, while the crosswind filter is applied as defined by Eq. (1), but with all nine sonics rather than five. These two-dimensional "test" filters are denoted as $3 \times 9,5$ $\times 9$, and $9 \times 9$, where the first and second integer in each pair correspond to the number of samples in the streamwise and crosswind quadratures, respectively.

The aliasing errors are determined by comparing results calculated with the low-resolution streamwise quadratures to those calculated with a high-resolution "reference" quadrature that uses all of the data available at the finer streamwise resolution $U_{c} t_{s}$. The number of data points in the high-resolution streamwise quadrature depends on $\Delta_{f}$ and $U_{c}$, and thus varies with configuration and meteorology, but is roughly on the order of 100 samples. The high-resolution quadrature is implemented by applying (6) in wavenumber space, using (8) with $\Delta_{f}=8 S \cos \Theta$, and then recovering the filtered time series by means of an inverse Fourier transform. The two-dimensional reference filter is labeled $\mathrm{hr} \times 9$, where hr denotes high-resolution; the crosswind quadrature for the reference filter is again a nine-sonic tophat filter. Figure 3 compares the streamwise response functions of both the low-resolution test filters and the high-resolution reference filter. As would be expected, increasing the number of points in the low-resolution quadratures clearly improves their match to the highresolution quadrature.

Figure 4 shows 1D streamwise power spectra of the

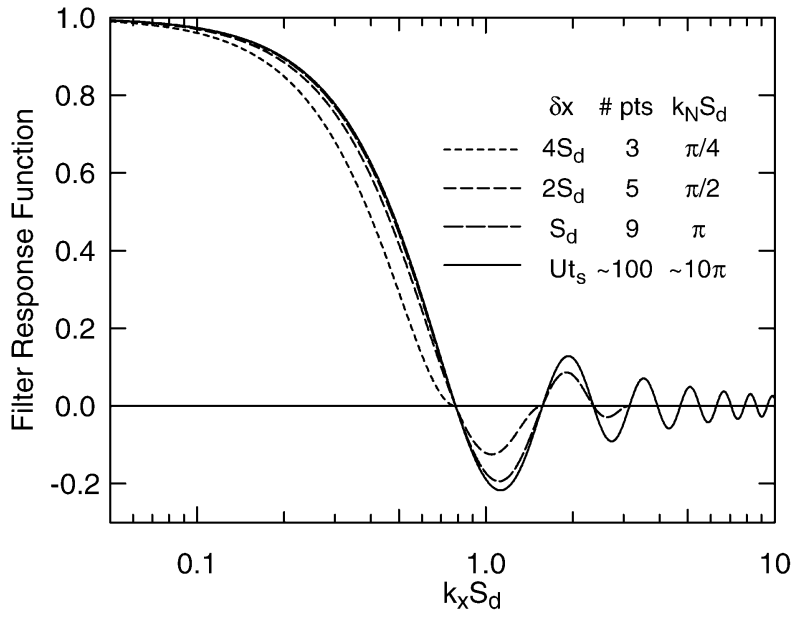

FIG. 3. Response functions of top-hat test and reference filters used to examine crosswind aliasing. The discrete streamwise filters have width $\Delta_{f}=8 S_{d}$ and, as tabulated in the legend, are each composed of the number of points, \# pts, with spacing $\delta x ; k_{N} S_{d}$ are the Nyquist wavenumbers of the filters, normalized by $S_{d}$.

vertical velocity, $E_{w}$, for two cases with different meteorology and from different sonic configurations. The plots for both cases include the spectrum of the unfiltered velocity $w$, as well as the spectra of $\tilde{w}_{3 \times 9}, \tilde{w}_{5 \times 9}$, $\tilde{w}_{9 \times 9}$, and $w_{h r \times 9}$. The abscissa on these plots is the streamwise wavenumber $k_{x}$ normalized by sonic spacing; the Nyquist wavenumbers of the three-, five-, and nine-point streamwise quadratures occur at $k_{x} S_{d}=\pi /$ $4, \pi / 2$ and $\pi$, respectively. Figure 4 a shows data from the configuration with the smallest sonic spacing, $S_{d}=$ $0.5 \mathrm{~m}$, and for moderately unstable stratification, $z / L=$ -0.41 . For this case the Nyquist wavenumbers of the three low-resolution quadratures are all greater than the wavenumber of the spectral peak of the unfiltered velocity, and thus are in a wavenumber range where the spectral energy is decreasing with increasing wavenumber. As a consequence, there is minimal aliasing and the three low-resolution quadratures produce values for $\tilde{w}$ that are very similar to those for $\tilde{w}_{h r \times 9}$. The spectra of $\tilde{w}$ have systematic differences above $k_{x} S_{d} \sim 0.5$, corresponding to differences in the transfer functions for the different quadratures, but the contribution of this high-wavenumber band to $\tilde{w}$ is negligible.

Figure $4 \mathrm{~b}$ shows data from the configuration with the largest sonic spacing, $S_{d}=3.35 \mathrm{~m}$, and for weak stable stratification, $z / L=0.05$. For this case, the Nyquist wavenumbers of the low-resolution quadratures are less than or near the wavenumber of the spectral peak of $w$. As a consequence, there is significant aliasing associated with the low-resolution quadratures, which is evident by comparison of their spectra with the spectrum of $\tilde{w}_{\text {hr } \times 9}$. As expected, the amount of aliasing increases as the sample spacing of the quadrature increases and its Nyquist wavenumber correspondingly decreases.

One perhaps unexpected aspect of Fig. 4 is that the power spectra for the resolved-scale velocities do not 

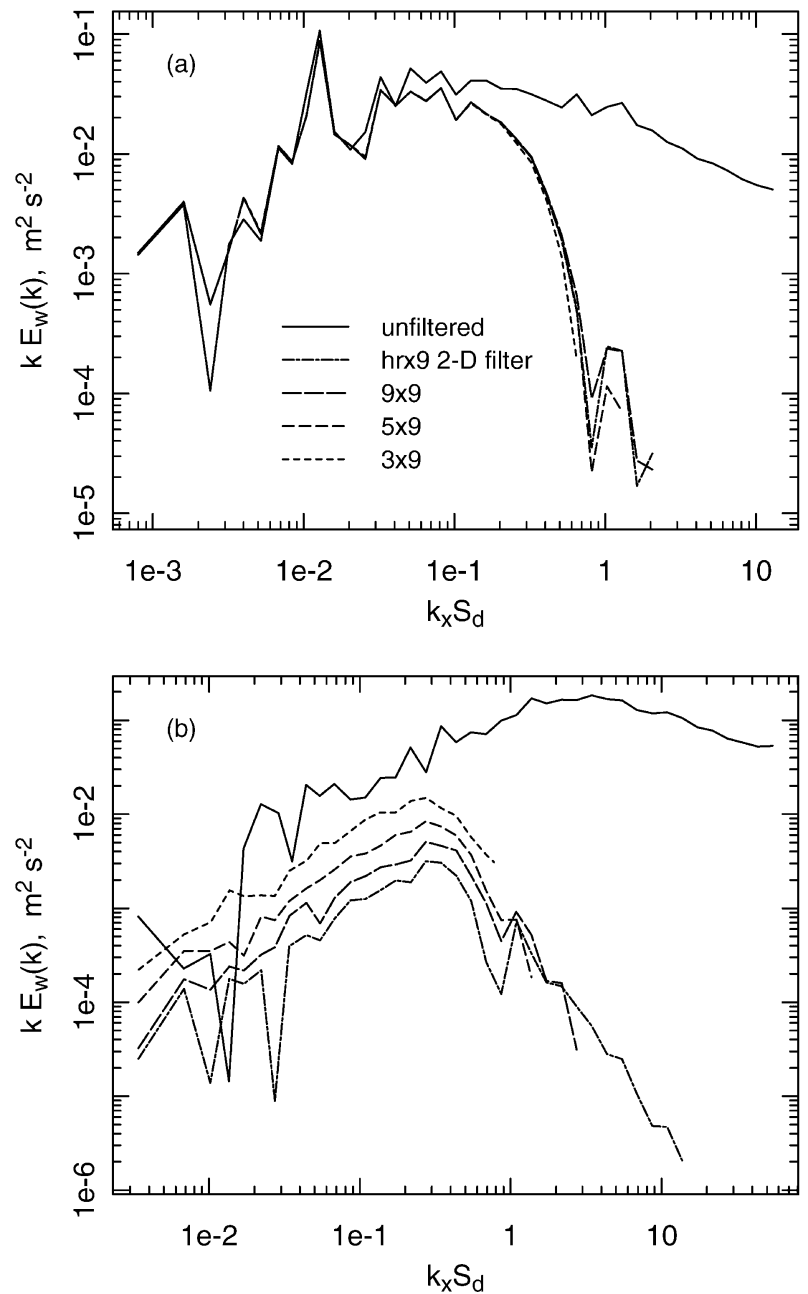

FIG. 4. Power spectra of unfiltered and 2D-filtered vertical velocity, $\Delta_{f}=8 S_{d}$ : (a) 1515 PDT $28 \mathrm{Sep} 2000, S_{d}=0.5 \mathrm{~m}, z / L=-0.41$, $\Lambda_{w} / \Delta_{f}=7.13$; (b) 0245 PDT 4 Sep 2000, $S_{d}=3.35 \mathrm{~m}, z / L=0.05$, $\Lambda_{w} / \Delta_{f}=0.29$

converge to the unfiltered spectra for $k_{x} \Delta_{f} \cong 8 k_{x} S_{d} \ll$ 1 . For the case shown in Fig. $4 \mathrm{a}, E_{\tilde{w}}$ more closely matches the behavior that might be expected from application of a low-pass filter: the filtered spectrum overlies the unfiltered spectrum at small wavenumbers. The reason that $E_{\tilde{w}}$ is not identical to $E_{w}$ at the smallest wavenumbers, even in Fig. $4 \mathrm{a}$, is that $\tilde{w}$ has been filtered in two dimensions. The resolved-scale velocity contains only Fourier components which have small wavenumbers in both the streamwise and crosswind directions. At each value of $k_{x}$, the one-dimensional streamwise spectra contain energy from all values of $k_{y}$, and the crosswind filter attenuates the energy for Fourier components with large $k_{y}$. This is more pronounced for the case shown in Fig. $4 \mathrm{~b}$ because the peak in the spectrum occurs at a larger value of $k_{y} \Delta_{f}$ than for the case in Fig. $4 \mathrm{a}$. This behavior has also been noted and discussed by Tong et al. (1999).

The aliasing errors of the low-resolution quadratures

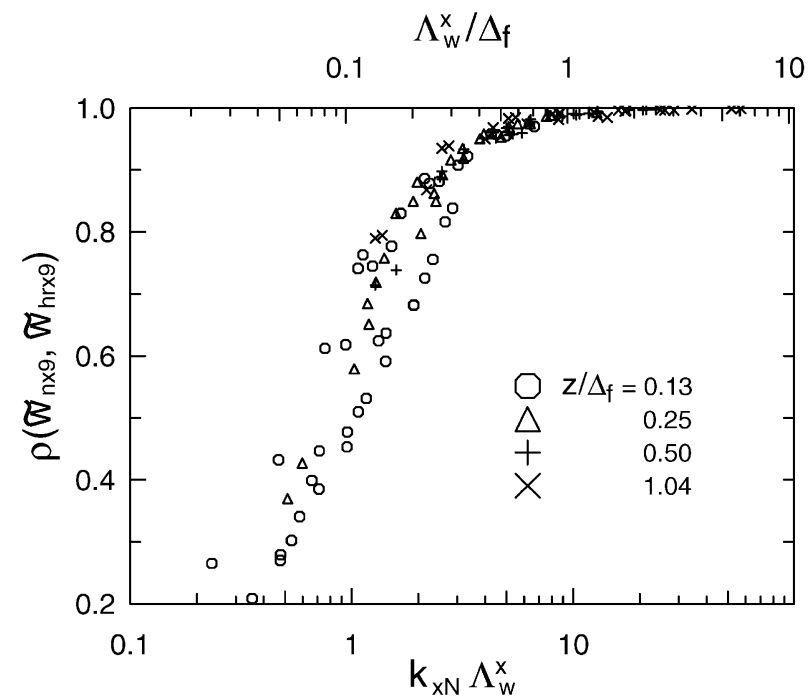

FIG. 5. Correlation coefficients $\rho\left(\tilde{w}_{n \times 9}, \tilde{w}_{\text {hr } \times 9}\right), n=\{3,5,9\}$, as a function of $\mathrm{k}_{x N} \Lambda_{w}^{x}$ and $\Lambda_{w}^{x} / \Delta_{f}=\Lambda_{w}^{x} / 4 S_{d}$. Different symbols are used for the data from each of the four sonic configurations, which are labeled with nominal values of $z / \Delta_{f}$, i.e., $\Delta_{f}=8 S_{d}$.

can be summarized by the correlation coefficients of $\tilde{w}_{3 \times 9}, \tilde{w}_{5 \times 9}$, and $\tilde{w}_{9 \times 9}$, with respect to $\tilde{w}_{h r \times 9}$, for example, $\rho\left(\tilde{w}_{5 \times 9}, \tilde{w}_{h r \times 9}\right)$. Figure 5 shows $\rho$ as a function of $k_{x N} \Lambda_{w}^{x}$, where $k_{x N}$ are the streamwise Nyquist wavenumbers of the low-resolution quadratures and $\Lambda_{w}^{x}$ is the wavelength of the peak of the streamwise vertical velocity spectrum. (Appendix A discusses estimation of the wavelengths of the spectral peaks.) The correlation coefficients in Fig. 5 are calculated from 41 stationary, 25-60 min data periods with wind directions within $\pm 33^{\circ}$ of the array normal, which were selected to cover a wide range of atmospheric stability for each of the four sonic configurations. The correlation coefficients collapse to a nearly universal relation dependent only on $k_{x N} \Lambda_{w}^{x}$, and there appears to be little or no residual dependence of $\rho$ on other independent variables such as atmospheric stability, height, or filter width. It may be concluded that the aliasing errors due to the finite sonic spacing are negligible $(\rho>0.9)$ when $k_{x N} \Lambda_{w}^{x}>3$.

The correlation coefficient is generally not sufficient to quantify the agreement between two time series, since they could have a correlation coefficient of unity but still differ by either an additive or multiplicative constant. This possibility is examined by also calculating the linear regression between each of the low-resolution quadratures and the reference quadrature (not shown). The intercepts of the linear regressions are negligible in all cases, and the slopes of the linear regressions increase toward unity as the correlation coefficient approaches unity. In particular, the linear regression of $\tilde{w}_{5 \times 9}$ on $\tilde{w}_{h r \times 9}$ is found to have a slope between 0.95 $(0.9)$ and 1.0, when the correlation coefficient between the two quadratures exceeds $0.8(0.6)$.

If it could be assumed that the crosswind and stream- 
wise power spectra had a similar shape and differed only in the wavelengths of their spectral peaks, then the aliasing errors of the low-resolution crosswind quadratures could be inferred from Fig. 5 by equating the independent variable $k_{x N} \Lambda_{w}^{x}$ with $k_{y N} \Lambda_{w}^{y}$. Atmospheric observations of crosswind spectra are limited to daytime aircraft data, which generally find that (frequency multiplied) crosswind spectra have sharper peaks than streamwise spectra and consequently have less lowwavenumber energy (e.g., Nicholls and Readings 1981). However, the significance of these spectral differences for aliasing by the low-resolution quadratures is not obvious. Further, similar comparisons of spectra are not available within the first $10 \mathrm{~m}$ above the surface, nor for stable stratification where the greatest aliasing occurs. As an alternative to comparisons of the spectra, the differences between aliasing by low-resolution quadratures in the streamwise and crosswind directions have been investigated directly by calculating $\tilde{w}_{9 \times 5}$ (corresponding to a five-point quadrature in the crosswind direction), $\tilde{w}_{5 \times 9}$ (corresponding to a five-point quadrature in the streamwise direction), and $\tilde{w}_{9 \times 9}$. All three filters again use trapezoidal, top-hat quadratures of width $8 S_{d} \cos \Theta$ in both the streamwise and crosswind directions. The differences between low-resolution quadratures in the streamwise and crosswind directions are estimated by comparing $\rho\left(\tilde{w}_{5 \times 9}, \tilde{w}_{9 \times 9}\right)$, plotted versus $k_{x N} \Lambda_{w}^{x}$, to $\rho\left(\tilde{w}_{9 \times 5}, \tilde{w}_{9 \times 9}\right)$, plotted versus $k_{y N} \Lambda_{w}^{y}$. (Appendix A also discusses estimates of $\Lambda_{w}^{y}$.) The correlation coefficients corresponding to the low-resolution quadrature in the crosswind direction are found to be systematically higher than those for the low-resolution quadrature in the streamwise direction (comparison not shown), but these differences are no greater than the overall spread of the data in Fig. 5. Thus aliasing errors by the discrete crosswind filters are perhaps overestimated by a small amount from Fig. 5 .

Sullivan et al. (2003) show that many statistics of the spatially filtered turbulence structure are principally a function of $\Lambda_{w}^{x} / \Delta_{f}=\Lambda_{w}^{x} / 4 S_{d}=k_{y N} \Lambda_{w}^{x} / 4 \pi$. In order to place their results in perspective with respect to the aliasing errors shown in Fig. 5, estimates of $\Lambda_{w}^{x} / \Delta_{f}$ are shown with a second abscissa at the top of the figure. Assuming that $k_{y N} \Lambda_{w}^{y} \cong k_{x N} \Lambda_{w}^{x}$, this scale is calculated as

$$
\frac{\Lambda_{w}^{x}}{\Delta_{f}}=\frac{\Lambda_{w}^{x}}{\Lambda_{w}^{y}} \frac{k_{y N} \Lambda_{w}^{y}}{4 \pi} \cong \frac{\Lambda_{w}^{x}}{\Lambda_{w}^{y}} \frac{k_{x N} \Lambda_{w}^{x}}{4 \pi} \cong \frac{k_{x N} \Lambda_{w}^{x}}{3 \pi},
$$

where the last equality in Eq. (12) assumes a constant value of $3 / 4$ for $\Lambda_{w}^{y} / \Lambda_{w}^{x}$. (As seen in Fig. A2, $\Lambda_{w}^{y} / \Lambda_{w}^{x}=$ $3 / 4$ corresponds roughly to its value for near-neutral to stable stratification, the stability range in which $\rho$ is significantly less than unity.)

The correlation coefficients between low- and highresolution quadrature estimates of $\tilde{u}, \tilde{v}$, and $\tilde{\theta}$ (not shown) are in all cases significantly higher than those shown in Fig. 5 for $\tilde{w}$. In most cases they exceed 0.95

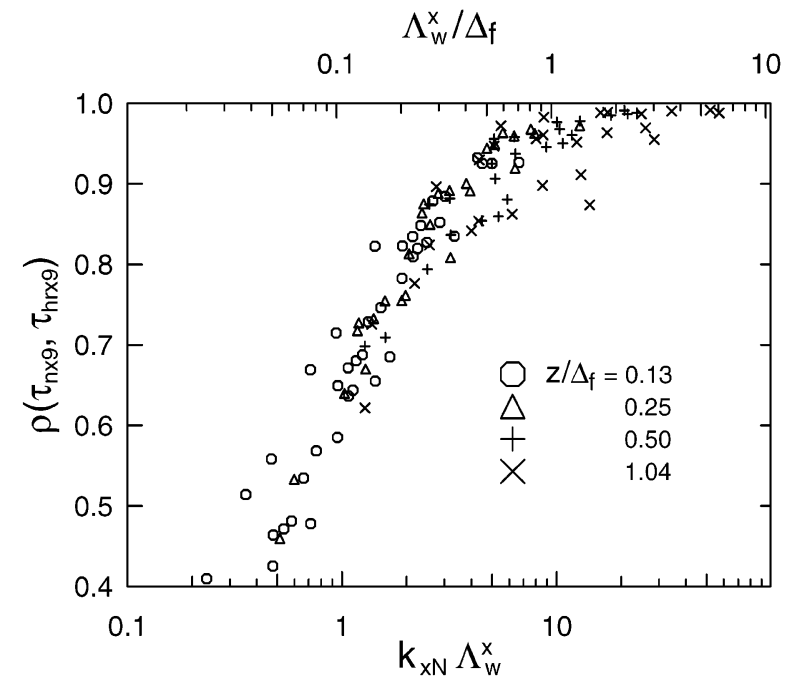

FIG. 6. Correlation coefficients $\rho\left(\tau_{n \times 9}, \tau_{h r \times 9}\right), n=\{3,5,9\}$, for $\tau_{13}$ as a function of $k_{x N} \Lambda_{w}^{x}$ and $\Lambda_{w}^{x} / \Delta_{f}$. See Fig. 5 for additional details.

and, for cases where the correlation coefficients for $\tilde{w}$ exceed 0.5 , they are generally no less than 0.98 , indicating that aliasing errors are minimal for those variables. Similar results were found by TWKB, albeit for a much narrower range of conditions. This happens because the peaks of the temperature and horizontal velocity spectra occur at wavelengths considerably larger than those for the vertical velocity spectra. For stable stratification, Kaimal et al. (1972; see also Kaimal and Finnigan 1994, their Fig. 2.9) find that $\Lambda_{v}^{x}$ is about twice $\Lambda_{w}^{x}$, while $\Lambda_{\theta}^{x}$ and $\Lambda_{u}^{x}$ are 3-5 times $\Lambda_{w}^{x}$.

Correlation coefficients between low- and high-resolution quadratures have also been calculated for the subfilter-scale velocities and temperature. The dependence of the correlation coefficients for these SFS variables on $k_{x N} \Lambda_{w}^{x}$ (not shown) is qualitatively similar to the behavior of the resolved-scale variables. This is not surprising, since the SFS velocities and temperature depend linearly on the corresponding resolved-scale variables, Eq. (3). Moreover, the SFS correlation coefficients for the cases shown in Fig. 5 are never less than 0.97. This would be expected for the cases where the resolved-scale correlation coefficients are themselves close to unity, $\Lambda_{w}^{x} / \Delta_{f}>1$. However, it can also be easily explained for the opposite limit, $\Lambda_{w}^{x} / \Delta_{f} \Rightarrow 0$, because then the SFS encompasses the energy-containing eddies, $\tilde{u}_{i}$ and $\tilde{\theta}$ approach their ensemble averages, and the SFS velocities and temperature are insensitive to errors in estimating the (negligible) resolved-scale turbulence.

Finally, correlation coefficients between low- and high-resolution quadratures have been calculated for the SFS second moments such as the stress tensor $\tau_{i j}$ and the temperature flux $q_{i} \equiv \widetilde{u_{i}} \theta-\tilde{u}_{i} \tilde{\theta}$. Figure 6 shows the correlation coefficients for $\tau_{13}$, the stress component commonly of most interest. They are quite similar to those for $\tilde{w}$ shown in Fig. 5. The correlation coefficients 
for $\tau_{22}$ and $q_{3}$ are comparable to those for $\tau_{13}$, while the correlation coefficients for $\tau_{23}$ are generally smaller. The correlation coefficients for the remaining SFS second moments are all somewhat higher than those shown in Fig. 6, with the highest values occurring for $q_{1}$ and the SFS temperature variance $\widetilde{\theta} \theta-\tilde{\theta} \tilde{\theta}$.

\section{Surrogate streamwise filtering using Taylor's hypothesis}

Taylor's hypothesis (Taylor 1938) is frequently used in turbulence research to convert a time series at a fixed point in space to a spatial signal at a fixed instant in time. Past work has focused on quantifying the validity of Taylor's hypothesis for interpreting measurements of energy spectra (Lumley 1965; Wyngaard and Clifford 1977). Here, the validity of Taylor's hypothesis is first examined using data from the sonic anemometers on HATS towers $a$ and $b$, which were displaced in the streamwise direction as described in section 2a. These data can be used to find the eddy advection velocity $U_{c}$ for unfiltered variables. However, since the time series data from a single sonic anemometer cannot be filtered in the lateral direction, they cannot address the validity of Taylor's hypothesis for determining filtered turbulence statistics and subfilter stresses. Therefore, this issue will be examined with numerical LES results using spatial and temporal filtering at a scale larger than the simulation grid size. Specifically, SFS stresses determined from purely spatial horizontal filtering are compared to SFS stresses obtained from a combination of streamwise time filtering and crosswind spatial filtering.

\section{a. Eddy advection velocity from spatial cross correlations}

The spatial cross correlation of velocity signals is a useful tool for examining the validity of Taylor's hypothesis and for determining the eddy advection velocity (Powell and Elderkin 1974). As illustrated in Hinze (1959, p. 422), Taylor's hypothesis suggests a peak in the cross correlation at a time lag $d t=d x / U$, where $d x$ is the distance at which the cross correlation is evaluated and $U$ is the mean streamwise velocity. If Taylor's hypothesis holds exactly, $\rho_{i}(d x, d t) \equiv\left\langle u_{i}^{\prime}(x, t) u_{i}^{\prime}(x+d x\right.$, $t+d t)\rangle /\left\langle u_{i}^{\prime 2}\right\rangle=1$ at $d t=d x / U$. Here, $u_{i}^{\prime}$ denotes a deviation from the ensemble average, $u_{i}^{\prime} \equiv u_{i}-\left\langle u_{i}\right\rangle$.

Figure 7 shows representative values of spatial cross correlations for the streamwise wind component $u_{1}$ as a function of time lag, obtained from the sonic data at two heights on HATS towers a and $\mathrm{b}$. The time lag $d t$ is normalized by $d x_{\text {eff }} / U$, where the effective tower displacement $d x_{\text {eff }} \equiv d x \cos \Theta$ corrects the actual displacement $d x$ for deviation of the wind from a direction parallel to the tower separation. To a first approximation, the results show that the peak correlation indeed occurs near $d t \approx d x_{\mathrm{eff}} / U$, with the maximum correlation increasing with height. More precisely, however, the

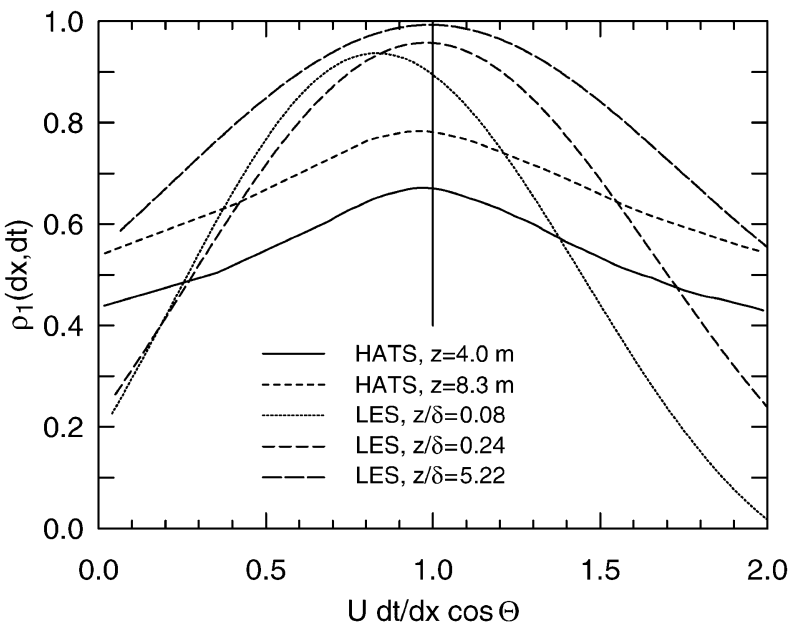

FIG. 7. Spatial cross correlations $\rho$ for the streamwise wind component $u_{1}$ as a function of normalized time lag. For the HATS data, $d x=17.3 \mathrm{~m}$, and for the LES data, $d x=\delta=52.4 \mathrm{~m} ; \delta$ is the LES horizontal mesh spacing.

cross-correlation peak occurs slightly left of the vertical line at $U d t / d x_{\text {eff }}=1$. Defining the eddy advection velocity as $U_{c} \equiv d x_{\text {eff }} / d t_{\max }$, where $d t_{\max }$ is the time lag at the maximum correlation, the results in Fig. 7 imply that $U_{c}$ is slightly higher than the mean wind speed $U$. Note that the advection velocity is determined here from the unfiltered velocity field and thus incorporates effects from all wavenumbers. While it is known that the advection velocity depends on the scales of motion [see e.g., Fisher and Davies (1963), who analyzed bandpassfiltered signals], we expect the present results to be associated with the energy-containing scales that predominantly determine the peak of the cross correlation.

Figure 7 also includes spatial cross correlations obtained from the LES results described in the next section. The LES cross correlations differ conceptually from those obtained with the field data in that they are based on filtered, resolved-scale eddies; results are shown for three values of $z / \delta$, the ratio of height to the filter width $\delta$ used to generate the LES flow field. The ratio $U_{c} / U>1$ for all three heights and decreases with height as found previously by Powell and Elderkin (1974). For both the field data and the LES results, the maximum cross correlation increases with height, perhaps reflecting the shift in turbulence to larger scales with increasing height. The maximum cross correlation is greater for the LES results than for the field data, presumably because the LES results only include a fraction of the total turbulence.

The cross-correlation analysis of Fig. 7 was applied to the HATS field data from forty-seven 27-min nearneutral periods with $|\Theta|<15^{\circ}$. The wind direction limit is based on finding that the peak correlation value decreases with increasing $|\Theta|$ for $|\Theta|>15^{\circ}$. Clearly, if the wind direction is not parallel to the line between the two towers, the two sonics do not sample exactly 
TABLE 2. Quartiles of $R=U_{c} / U$ and the peak cross-correlation $\rho^{\max }$ from HATS data.

\begin{tabular}{|c|c|c|c|c|c|c|}
\hline \multirow{2}{*}{$\begin{array}{l}\text { Height } z_{\mathrm{AGL}}-d_{0}(\mathrm{~m}) \\
\text { Velocity component, } i\end{array}$} & \multicolumn{3}{|c|}{4.01} & \multicolumn{3}{|c|}{8.34} \\
\hline & 1 & 2 & 3 & 1 & 2 & 3 \\
\hline$R_{i}^{\mathrm{q} 1}$ & 1.06 & 0.97 & 0.98 & 1.06 & 0.94 & 1.01 \\
\hline$R_{i}^{\text {med }}$ & 1.10 & 1.03 & 1.03 & 1.11 & 0.98 & 1.03 \\
\hline$R_{i}^{\mathrm{q} 3}$ & 1.22 & 1.04 & 1.09 & 1.16 & 1.00 & 1.08 \\
\hline$\rho_{i}^{l \max }$ & 0.60 & 0.56 & 0.14 & 0.74 & 0.69 & 0.31 \\
\hline
\end{tabular}

the same air parcels, thus decreasing the spatial cross correlation even if the turbulence is frozen. Table 2 shows, for two heights and each velocity component $i$, the first quartile (q1), median (med), and third quartile (q3) of the ratio $R_{i} \equiv U_{c} / U$, as well as the average value of the peak in the correlation $\left(\rho_{i}^{\max }\right)$. The turbulence intensity, TI $\equiv \sigma_{1} / U$, where $\sigma_{1}$ is the rms streamwise velocity, was also calculated for each data period, but $U_{c} / U$ does not appear to be dependent on TI.

Systematic differences in $R_{i}$ are observed for the three velocity components: $R_{1}>R_{3}>R_{2}$, in agreement with Powell and Elderkin (1974). The spread around these mean values, $\left(R_{i}^{q 3}-R_{i}^{q 1}\right) / 2$, is about 0.04 . The maximum of the spatial cross-correlation $\rho_{i}^{\max }$ is also dependent on the velocity component, $\rho_{1}^{\max }>\rho_{2}^{\max } \gg \rho_{3}^{\max }$, and increases with height. Thus the measured advection velocity may be up to $10 \%-20 \%$ larger than the mean velocity. Unfortunately, the dependence on velocity component, as well as a possible dependence on wavenumber, complicates use of the measured $U_{c}$ in our surrogate spatial filtering. We can only note that streamwise filter widths may be greater and streamwise gradients smaller than estimated from time series data on the basis of the mean wind speed.

\section{b. Taylor's hypothesis for SFS stress}

The accuracy of Taylor's hypothesis for SFS stress measurements was first examined by Murray et al. (1996) using direct numerical simulation of low Reynolds number channel flow. TWKB used an LES of a moderately convective boundary layer in order to estimate filter performance for high Reynolds number atmospheric data. The latter computed resolved-scale velocities and SFS stresses using a spectral-cutoff filter with two cutoff wavenumbers, $k_{c} z=1.68$ and 0.84 , equal to $1 / 5$ and $1 / 10$ of the LES cutoff wavenumber, respectively. Using $k_{c}=\pi / \Delta_{f}$, these scales correspond to $z / \Delta_{f}=0.53$, and $z / \Delta_{f}=0.27$. They found high correlations between the $2 \mathrm{D}$ spatially filtered velocity $\tilde{u}$ and the surrogate velocity $\tilde{u}^{\mathrm{T}}$ obtained with streamwise time filtering and crosswind spatial filtering: $\rho\left(\tilde{u}, \tilde{u}^{\mathrm{T}}\right) \sim$ 0.9 for both values of $z / \Delta_{f}$, but lower correlations for the true and surrogate SFS stresses, $\rho\left(\tau_{13}, \tau_{13}^{\mathrm{T}}\right) \sim 0.7$, for $z / \Delta_{f}=0.53$.

TWKB's analysis of LES turbulent fields is extended here to a wider range of the parameter $z / \Delta_{f}$, thus also considering the effects of increasing isotropy of tur- bulence and decreasing turbulence intensity with height. Moreover, the present analysis uses filters that mimic closely those used in our analysis of the HATS data, specifically a Gaussian filter in the streamwise direction and a top-hat filter in the crosswind direction. In the following, the SFS stresses $\tau_{i j}$ and $\tau_{i j}^{\mathrm{T}}$ are compared by means of both correlation coefficients and mean-square errors.

Details of the LES model and simulation parameters used in the analysis are presented in appendix B. In order to obtain a "temporal" field for applying Taylor's hypothesis, we choose a vertical reference plane transverse to the mean flow located at the center of the computational domain, $x=x_{0}$. A horizontal line on this plane mimics a crosswind array of sensors placed at height $z$. The velocities $u_{i}\left(x_{0}, y, z, t\right)$ are used with Taylor's hypothesis to obtain a surrogate spatial data distribution $u_{i}^{\mathrm{T}}\left(x, y, z, t_{0}\right) \equiv u_{i}\left(x_{0}, y, z, t\right)$, where $x=$ $x_{0}-U_{c}\left(t-t_{0}\right)$. The advection velocity $U_{c}$ is equated to the mean velocity of the spatial field averaged over the $x$ direction, $U_{c}(y, z, t)=\left\langle u_{1}(x, y, z, t)\right\rangle_{x}$. Note that scales below the LES filter scale $\delta$ are not explicitly considered in this analysis; they are only accounted for implicitly by the SFS parameterization. The sensitivity to the ratio $\Delta_{f} / \delta$ is examined by repeating the analysis for two filter sizes, $\Delta_{f}=4 \delta$ and $8 \delta$, or $\Delta_{f} / H=0.21$ and 0.42 where the height of the computational domain $H$ is the effective boundary layer height. While these values of $\Delta_{f} / H$ are significantly larger than those of the HATS data, the simulation analysis coincides with the field measurements in terms of $z / \Delta_{f}$.

Correlation coefficients between time series of $\tau_{i j}$ and $\tau_{i j}^{\mathrm{T}}$ were computed for all horizontal planes and the 11 , 13, and 33 stress components. The correlation coefficients, averaged in the $y$ direction, are plotted as a function of $z / \Delta_{f}$ in Fig. 8. For $z / \Delta_{f}>0.25, \rho\left(\tau_{i j}, \tau_{i j}^{\mathrm{T}}\right)$ are greater than 0.98 for all three stress components, and even at the lowest grid point, $z / \Delta_{f}=0.02$, the correlations are still large (0.89). There are no significant differences between the results for $\Delta_{f} / \delta=8$ and $\Delta_{f} / \delta$ $=4$. The remainder of the analysis will concentrate on $\tau_{13}$, since determination of the momentum flux is often of primary interest. From Fig. 8, it appears that correlations calculated for $\tau_{13}$ may be a reasonable estimate for $\tau_{33}$ and a conservative estimate for $\tau_{11}$.

Examination of the terms contributing to $\tau_{13} \equiv \widetilde{u_{1} u_{3}}$ $-\tilde{u}_{1} \tilde{u}_{3}$ (not shown) finds that $\rho\left(\tilde{u}_{1}, \tilde{u}_{1}^{\mathrm{T}}\right)>0.995$ and 


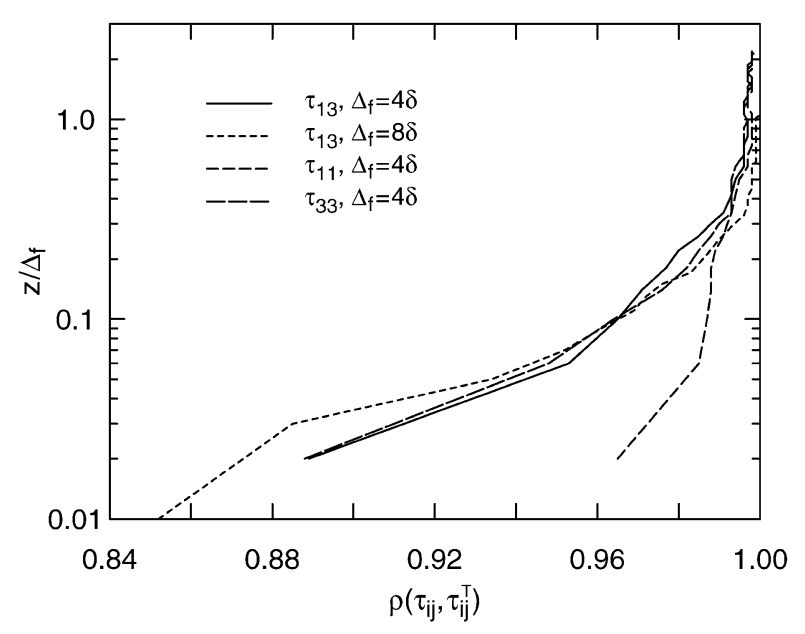

FIG. 8. Profiles of correlation coefficients between the SFS stresses determined with surrogate, $\tau_{i j}^{\mathrm{T}}$, and actual, $\tau_{i j}$, 2D spatial filtering. SFS stresses were calculated by explicit horizontal 2D filtering of the LES velocities at $\Delta_{f}=4 \delta$ and $8 \delta$.

$\rho\left(\tilde{u}_{3}, \tilde{u}_{3}^{\mathrm{T}}\right)>0.95$ for all $z / \Delta_{f}$, and $\rho\left(\widetilde{u_{1} u_{3}}, \widetilde{u_{1} u_{3}^{\mathrm{T}}}\right)$ is similar but slightly higher than $\rho\left(\tilde{u}_{3}, \tilde{u}_{3}^{\mathrm{T}}\right)$. These values are larger than those presented in TWKB. The reason appears to be that their 2D spectral-cutoff filter is less localized in physical space than our combination of top-hat and Gaussian spatial filters. Applying a 2D spectral-cutoff filter in our analysis produces results similar to TWKB.

It is of interest to also examine the dependence of the correlation coefficients on turbulence intensity. Although it was found that turbulence intensity does not influence the ratio of advection velocity to mean streamwise velocity, it has been considered a crucial parameter for the validity of Taylor's hypothesis (in unfiltered tur- bulence) by various investigators (Willis and Deardorff 1976; Wyngaard and Clifford 1977). Further, the values of $z / H$ in the field measurements are much lower than those of the simulation, while the turbulence intensity levels in the simulation and the field measurements are more comparable. The correlation coefficients are plotted as a function of turbulence intensity in Fig. 9. In the simulation, the turbulence intensity is computed from the rms of the resolved velocity, which should be compared to the turbulence intensity of the resolvedscale velocity in the field data. For near-neutral field data, the mean resolved-scale turbulence intensity was 0.137 with a standard deviation of 0.051 , that is, most of the data fall within the range $0.086<\mathrm{TI}<0.188$ highlighted in Fig. 9. In this range the correlation coefficient is larger than 0.95 , further supporting use of Taylor's hypothesis.

The correlation coefficient alone does not provide complete information about the level of agreement between two variables. In order to ensure that the magnitude of the stresses is also similar, the normalized square error, defined as $\epsilon(x, z)=\left\langle\left\langle\left(\tau_{13}-\tau_{13}^{\mathrm{T}}\right)^{2}\right\rangle_{t} /\left\langle\tau_{13}^{2}\right\rangle_{t}\right\rangle_{y}$ and the normalized rms error $\sqrt{\epsilon}$ are computed. These errors are $\epsilon \approx 0.06(\sqrt{\epsilon} \approx 0.24)$ at the smallest $z / \Delta_{f}$, decreasing to $\epsilon<0.01(\sqrt{\epsilon}<0.12)$ for $z / \Delta_{f}>0.25$.

\section{Spatial gradients of resolved-scale variables \\ a. Finite-difference response functions}

The HATS measurement arrays were designed to enable estimation of spatial gradients of resolved-scale velocities and temperature. The availability of nine sonics in the $d$ arrays enables use of a fourth-order centereddifference scheme to estimate crosswind gradients,

$$
\frac{d \tilde{\mathbf{u}}(y)}{d y}=\frac{\tilde{\mathbf{u}}\left(y+2 S_{e}\right)+8 \tilde{\mathbf{u}}\left(y+S_{e}\right)-8 \tilde{\mathbf{u}}\left(y-S_{e}\right)-\tilde{\mathbf{u}}\left(y-2 S_{e}\right)}{12 S_{e}},
$$

where $S_{e} \equiv S_{d} \cos \Theta$. Similarly, with the assumption of Taylor's hypothesis, a fourth-order centered difference can also be used to estimate the streamwise gradients. However, due to limited instrumentation resources, vertical gradients are estimated simply as

$$
\frac{d \tilde{\mathbf{u}}}{d z}=\frac{\tilde{\mathbf{u}}\left(z_{s}\right)-\tilde{\mathbf{u}}\left(z_{d}\right)}{z_{s}-z_{d}} .
$$

At the height $z_{d}$ where the horizontal gradients are evaluated, this is a less accurate first-order finite difference.

The response functions for the two finite-difference schemes are, respectively, $\sin \left(k S_{e}\right)\left[4-\cos \left(k S_{e}\right)\right] / 3 k S_{e}$ and $\sin (k \delta z) / k \delta z$, where $\delta z \equiv z_{s}-z_{d}$. These are plotted in Fig. 10 as a function of $k S_{e}$, along with the response functions of the discrete five-point top-hat filter used in the crosswind direction and the matched Gaussian filter used in the streamwise direction. Two response functions are shown for the vertical gradient: one for $\delta z / S_{e}$ $=1.03$, corresponding to the first sonic configuration, and the other for $\delta z / S_{e}=2$, corresponding to configurations 2-4. All response functions are truncated at their respective Nyquist wavenumbers.

The response functions of the finite-difference gradient estimates would ideally be unity throughout the wavenumber range of the resolved scales. In addition, it would be desirable to match the response functions for the horizontal and vertical derivatives. These goals are achieved moderately well for the fourth-order centered-difference scheme and for the vertical difference with $\delta z / S=1.03$. The performance is not as good for the vertical difference with $\delta z / S=2$. In retrospect, this can be improved in future experiments by matching the 


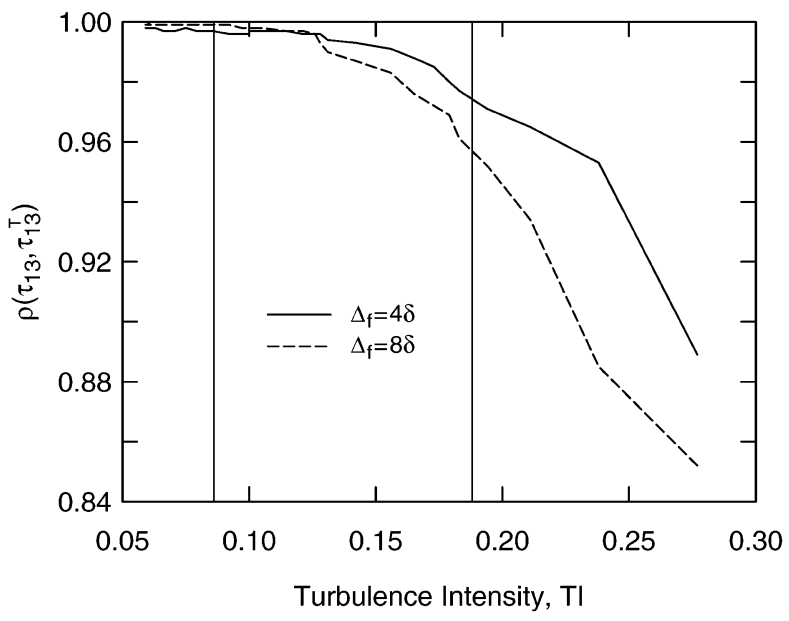

FIG. 9. The correlation coefficients of Fig. 8 as a function of turbulence intensity, TI $\equiv \sigma_{1} / U$. Typical values of turbulence intensity for HATS 2D-filtered velocities fall within the range $0.086<$ TI $<$ 0.188 shown by the two vertical lines.

transfer functions for the horizontal and vertical gradients when designing the sonic anemometer spatial arrays.

\section{b. Quality of finite-difference gradient estimates}

Aliasing is a particular concern for the spatial gradients, since the power spectra of the derivatives do not decrease at high wavenumbers, but rather increase as $k^{1 / 3}$. This issue has been examined by focusing again on the streamwise gradients, where detailed spectral analysis is possible. Figure 11 shows the (wavenumber multiplied) power spectra of $\partial \tilde{w} / \partial x$ for the same case shown in Fig. 4b, which exhibited considerable aliasing for $\tilde{w}$. The spectra of $\partial w / \partial x$ and $\partial \tilde{w}_{h r \times 9} / \partial x$ were obtained by multiplying the respective vertical velocity spectra by $k^{2}$, thus providing exact gradient spectra, while the gradients of low-resolution-filtered vertical velocities, for example, $\partial \tilde{w}_{3 \times 9} / \partial x$, were obtained using the fourth-order centered difference, Eq. (13). It is gratifying to note that the aliasing associated with the combination of lowresolution filtering and finite-difference gradient estimation is very similar to that seen in Fig. $4 \mathrm{~b}$ for just the low-resolution filtering. The reason for this is that finite-difference gradient estimation acts as an effective low-pass antialiasing filter, as can be seen from its response function, Fig. 10.

The quality of the finite-difference estimates of $\partial \tilde{\mathbf{u}} /$ $\partial x$ and $\partial \tilde{\theta} / \partial x$ is quantified by calculating the correlation coefficients with respect to $\partial \tilde{\mathbf{u}}_{h r \times 9} / \partial x$ and $\partial \tilde{\theta}_{h r \times 9} / \partial x$ calculated in wavenumber space. Figure 12 shows these correlation coefficients for $\partial \tilde{w} / \partial x$, again as a function of $k_{x N} \Lambda_{w}^{x}$. Several features can be noted in comparison to Fig. 5 for $\tilde{w}$. First, the correlation coefficient approaches unity more slowly at large values of $k_{x N} \Lambda_{w}^{x}$. The reason for this is that $\partial \tilde{w}_{h r \times 9} / \partial x$ has significant energy at large wavenumbers that is eliminated by the low-pass re-

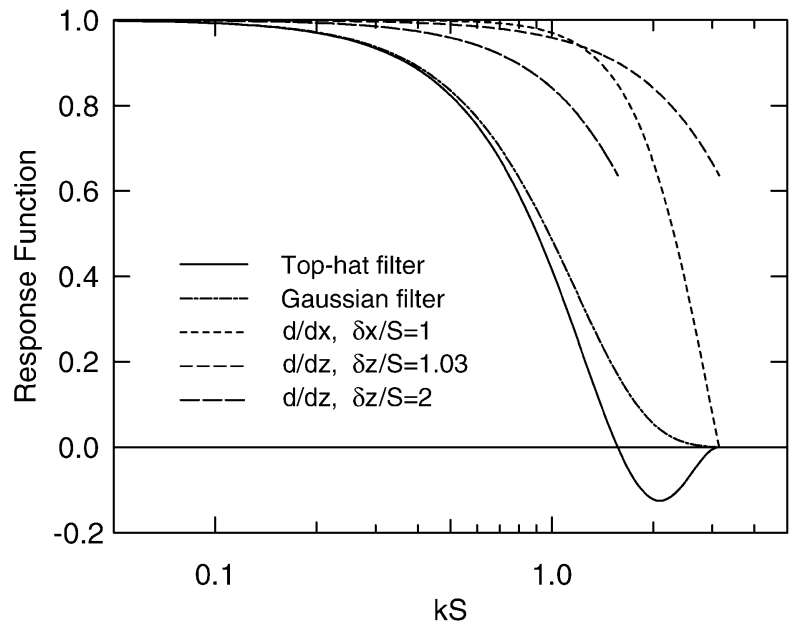

FIG. 10. Response functions for finite-difference estimates of spatial gradients. Here, $d / d x$ is computed with a first-order centereddifference scheme over a streamwise interval $\delta x ; d / d z$ is computed with a first-order centered-difference scheme over a height interval $\delta z$.

sponse of the finite-difference derivatives. Second, there is more scatter in the correlation coefficients. This appears to be caused by a sensitive balance between increased aliasing associated with the low-resolution quadrature and more effective antialiasing associated with a low-resolution finite difference. Finally, the correlation coefficients for $\partial \tilde{w} / \partial x$ decrease somewhat more slowly with decreasing $k_{x N} \Lambda_{w}^{x}$. Correlation coefficients for $\partial \tilde{u} / \partial x, \partial \tilde{v} / \partial x$, and $\partial \tilde{\theta} / \partial x$ are higher than those for $\partial \tilde{w} /$ $\partial x$ and are in most cases greater than 0.8 .

\section{Conclusions}

An extensive turbulence dataset has been collected with crosswind arrays of sonic anemometers deployed

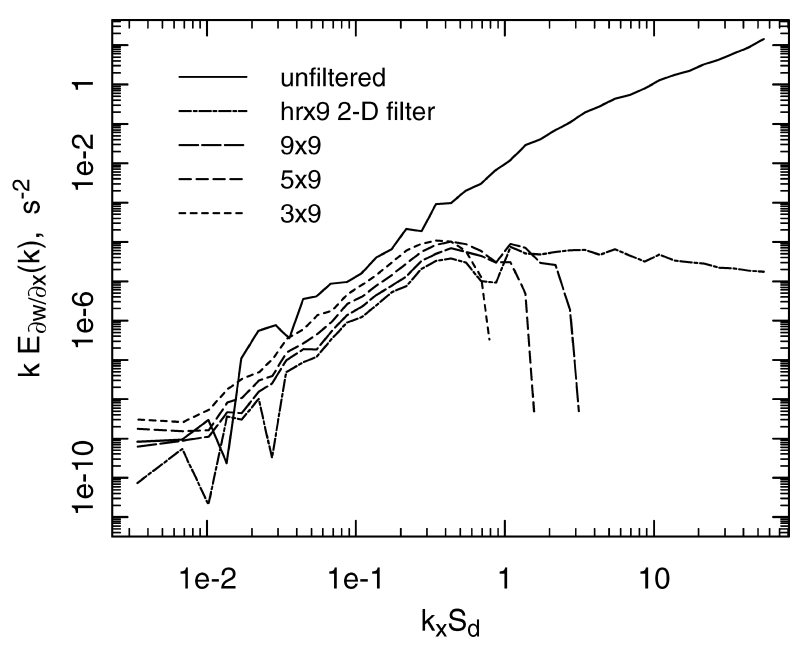

FIG. 11. Power spectra for streamwise gradients of unfiltered and $2 \mathrm{D}$-filtered vertical velocity, $\Delta_{f}=8 S_{d}$; conditions as listed for Fig. $4 \mathrm{~b}$. 


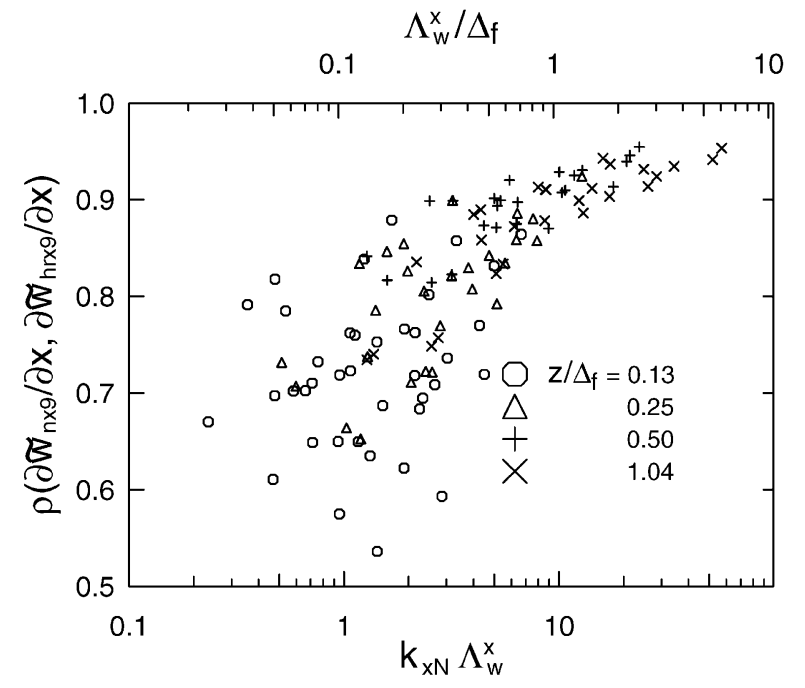

FIG. 12. Correlation coefficients $\rho\left(\partial \tilde{w}_{n \times 9} / \partial x, \partial \tilde{w}_{h r \times 9} / \partial x\right), n=\{3,5,9\}$, as a function of $k_{x N} \Lambda_{w}^{x}$ and $\Lambda_{w}^{x} / \Delta_{f}$. See Fig. 5 for additional details.

in the atmospheric surface layer. This field project was designed to investigate the relationships between spatially filtered and subfilter-scale turbulence. Initial results from those analyses are found in Sullivan et al. (2003) and Kleissl et al. (2003). The data are available for download at www.atd.ucar.edu.

The measurements were designed to enable $2 \mathrm{D}$ horizontal spatial filtering of the turbulence by averaging over five adjacent sonic anemometers in the crosswind direction and by using Taylor's hypothesis and the mean wind speed to interpret time averages as streamwise spatial averages. The approximation of crosswind filtering by averaging over five sonics with finite spacing $S$ was found to cause aliasing errors in the filtered turbulence. The aliasing errors depend on the relative magnitudes of the Nyquist wavenumber of the discrete crosswind sampling $k_{y N}=\pi / S$ and the wavelength at the peak of the crosswind power spectrum of vertical velocity $\Lambda_{w}^{y}$. As expected, the aliasing errors were found to decrease with increasing $k_{y N} \Lambda_{w}^{y}$. The aliasing errors are generally negligible for both resolved and SFS wind and temperature, with the exception of the resolvedscale vertical velocity $\tilde{w}$, which has nonnegligible aliasing errors for $k_{y N} \Lambda_{w}^{y}<3$. The aliasing errors for the SFS momentum and heat fluxes, $\tau_{13}$ and $q_{3}$, are similar to those for $\tilde{w}$.

The validity of Taylor's hypothesis for surrogate spatial filtering was examined for neutral stratification, both with measurements at two towers displaced in the streamwise direction and with LES-generated turbulence fields. Field data from sensors displaced in the streamwise direction suggest that the eddy advection velocity $U_{c}$ may be up to $10 \%-20 \%$ larger than the mean wind speed. Thus the width of the streamwise filter is underestimated (and streamwise gradients are overestimated) by using Taylor's hypothesis and the mean wind speed for surrogate spatial analysis. Both the eddy advection velocity and the maximum in the spatial cross correlation, which by Taylor's hypothesis should be unity, have a systematic dependence on the velocity component.

LES turbulence fields were used to calculate correlations between $\tau_{13}$, obtained by $2 \mathrm{D}$ spatial filtering, and $\tau_{13}^{\mathrm{T}}$, obtained by spatial filtering in the crosswind direction but using Taylor's hypothesis for surrogate streamwise filtering. The correlation between $\tau_{13}$ and $\tau_{13}^{\mathrm{T}}$, for $z / \Delta_{f}>0.25$ is found to be sufficiently large $(>0.98)$ to justify surrogate filtering. Note that for highly convective conditions, in which the resolved turbulence intensity increases, the accuracy of Taylor's hypothesis is expected to degrade further (Powell and Elderkin 1974).

The measurement array design also permits estimation of spatial gradients of filtered variables. Vertical gradients are estimated by a simple first-order difference, but horizontal gradients can be estimated by a fourth-order centered difference. The response function of the fourth-order centered difference is close to unity throughout the wavenumber range of the resolved scales. Moreover, the finite-difference horizontal gradient estimation also acts as an effective low-pass filter, so that the gradients have minimal additional aliasing in the crosswind direction beyond that already present in the spatially filtered variables.

Acknowledgments. The authors are grateful to William Eichinger, Fernando Porté-Agel, Scott Richardson, and John Wyngaard for the loan of sonic anemometers used in this project and to Chenning Tong for discussions both during the planning of the field measurements and during the subsequent data analysis. We gratefully acknowledge the dedicated and skillful work of the NCAR Atmospheric Technology Division staff who made these measurements possible: Kurt Knudson, Gordon Maclean, Charlie Martin, John Militzer, and Steve Semmer. We also thank Ceil Howe III for permitting these measurements to be made on land owned by Westlake Farms. Additional support for Peter Sullivan and Jeff Weil was provided, respectively, by the Office of Naval Research and the Army Research Office. The Johns Hopkins University participants were supported by Grant NSF-ATM 01300766.

\section{APPENDIX A}

\section{Estimation of the Wavelength at the Spectral Maximum}

In section $3 b$ of this paper, it is shown that the discrete quadrature of the crosswind filter produces aliasing errors whose magnitude depends both on the spatial separation of the samples that compose the discrete filter and on the wavelength of the spectral peak of the unfiltered velocity component. Our analysis of those aliasing errors requires values for both $\Lambda_{w}^{x}$ and $\Lambda_{w}^{y}$, the 
wavelengths of the peaks of the streamwise and crosswind spectra.

A direct estimate of $\Lambda_{w}^{x}$ is determined from the power spectrum of the vertical wind component. This is accomplished by fitting the observed spectrum with a model for the frequency-multiplied (or logarithmic) power spectrum

$$
\frac{f E_{w}(f)}{\sigma_{w}^{2}}=\frac{A(\mu) f / f_{m}}{\left[1+1.5\left(f / f_{m}\right)^{\mu}\right]^{5 / 3 \mu}} .
$$

Here $E_{w}(f)$ is the power spectral density, $f$ is frequency, and

$$
A(\mu)=\frac{\mu \Gamma(5 / 3 \mu) 1.5^{1 / \mu}}{\Gamma(1 / \mu) \Gamma(2 / 3 \mu)}
$$

is determined by the constraint $\int_{0}^{\infty} E_{w}(f) d f=\sigma_{w}^{2}$, the variance of the vertical velocity component. Both $f_{m}$, the frequency at the peak of the logarithmic power spectrum, and $\mu$, which controls the sharpness of the peak, are adjusted to minimize the sum of the absolute differences between the model and the vertical velocity spectrum averaged over all sonics in either the $d$ or $s$ arrays. Then by Taylor's hypothesis, $\Lambda_{w}^{x}=U_{c} / f_{m}$. For stable stratification, the spectral model with $\mu \approx 5 / 3$ provides very good fits to the observed vertical velocity spectra. For unstable stratification, the best fits between the model and observed spectra are found for $\mu$ on the order of 1, but the best-fit model spectra often appear to exhibit small systematic departures from the observed spectra. In the latter cases, the model spectrum is less than the observed spectrum for $f<f_{m}$, crosses the observed spectrum in the inertial range, and is higher than the observed spectrum as $f$ approaches its Nyquist value. This behavior appears to be caused by the asymmetry in the curvature of Eq. (A1) on each side of the spectral peak.

The number and spacing of the sonics in the spatial filter arrays are not adequate to estimate $\Lambda_{w}^{y}$ directly from the crosswind spectrum. As an alternative, the sonics in each of the spatial filter arrays are used to directly calculate the crosswind cross-correlation function $\rho\left[w(y) w\left(y+r_{y}\right)\right]$ at spatial lags equal to integer multiples of the crosswind sonic spacing, that is, $r_{y}=m_{d} S_{d}$ $\cos \Theta$ or $m_{s} S_{s} \cos \Theta, 0 \leq m_{d} \leq 8$, and $0 \leq m_{s} \leq 4$. With the assumption that the observed cross-correlation function can be closely approximated by

$$
\rho\left(r_{y}\right)=\exp \left(-r_{y} / \mathcal{L}^{y}\right),
$$

the crosswind Eulerian integral length scale $\mathcal{L}^{y}$ can be estimated from the (interpolated) spatial lag where $\rho\left(r_{y}=\mathcal{L}^{y}\right)=e^{-1}$. (Note that this assumption is not realistic in the inertial range, since it implies a spectral power law at high frequencies equal to $f^{-2}$ rather than $f^{-5 / 3}$.) Then, again assuming the exponential model for the cross-correlation function, $\Lambda^{y}=2 \pi \mathcal{L}^{y}$ (e.g., Kaimal and Finnigan 1994, p. 63). The number and spacing of the sonics in the crosswind filter arrays are adequate to

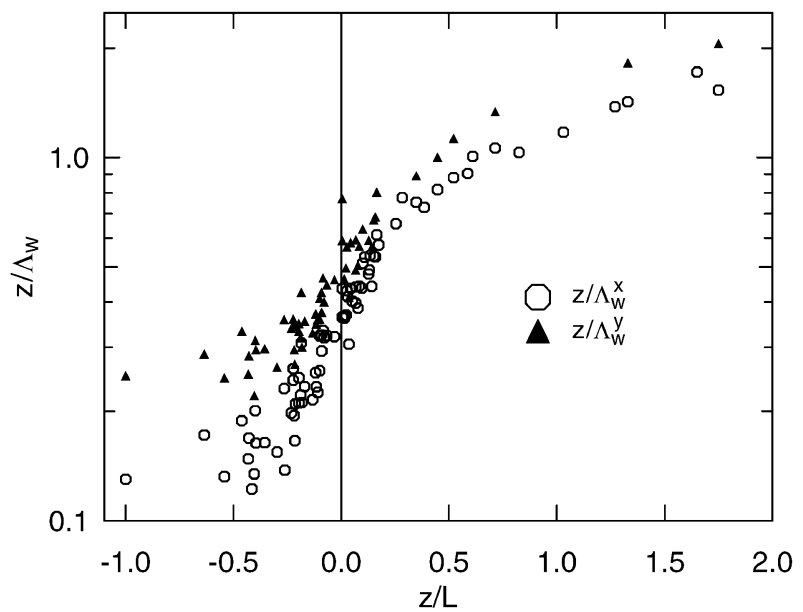

FIG. A1. Dependence of the wavelengths $\Lambda_{w}$ at the peaks of the streamwise and crosswind spectra of vertical velocity on atmospheric stability, $z / L$

estimate $\Lambda_{w}^{y}$ by this technique for all four array configurations, with the exception of stable stratification for the first configuration $\left(\mathcal{L}_{w}^{y} \ll S_{d}, S_{s}\right)$ and moderate to strongly unstable stratification for the $s$ array during the fourth configuration $\left(\mathcal{L}_{w}^{y}>4 S_{s}\right)$.

Values of $\Lambda_{w}^{x}$ have also been estimated by the exponential cross-correlation technique, using the time series data along with Taylor's hypothesis to estimate the streamwise cross-correlation function. The cross-correlation technique estimates of $\Lambda_{w}^{x}$ are found to agree with the direct spectral estimates generally within $\pm 10 \%$ for stable stratification and $\pm 15 \%$ for unstable stratification. The good comparison between the two techniques in the streamwise direction (not shown) provides confidence in the estimation of $\Lambda_{w}^{y}$ from the exponential cross-correlation technique. The increased scatter between the two estimates for unstable stratification is consistent with the increased uncertainty of the values of $\Lambda_{w}^{x}$ estimated directly from the unstable spectra.

Figure A1 shows exponential cross-correlation technique estimates of both $z / \Lambda_{w}^{x}$ and $z / \Lambda_{w}^{y}$ as a function of atmospheric stability, $z / L$. The data for $z / \Lambda_{w}^{x}$ are generally consistent with results for the horizontally homogeneous atmospheric surface layer reported by Kaimal et al. (1972, 1976, 1982) and Panofsky and Dutton (1984). Values of $\Lambda_{w}^{x}$ are found to exceed $\Lambda_{w}^{y}$ for all stabilities, as would be expected on account of the streamwise elongation of turbulent eddies by vertical shear within the surface layer (Nicholls and Readings 1981). Figure A2 shows $\Lambda_{w}^{y} / \Lambda_{w}^{x}$ as a function of $z / L$. The ratio of crosswind to streamwise wavelengths is about 0.78 for near-neutral and stable stratification and decreases sharply with increasing instability to a value approaching 0.55 for $z / L \approx-0.5$. In those cases where the crosswind wavelength cannot be estimated directly by the exponential cross-correlation technique, Fig. A2 is used to estimate $\Lambda_{w}^{y}$ from $\Lambda_{w}^{x}$. 


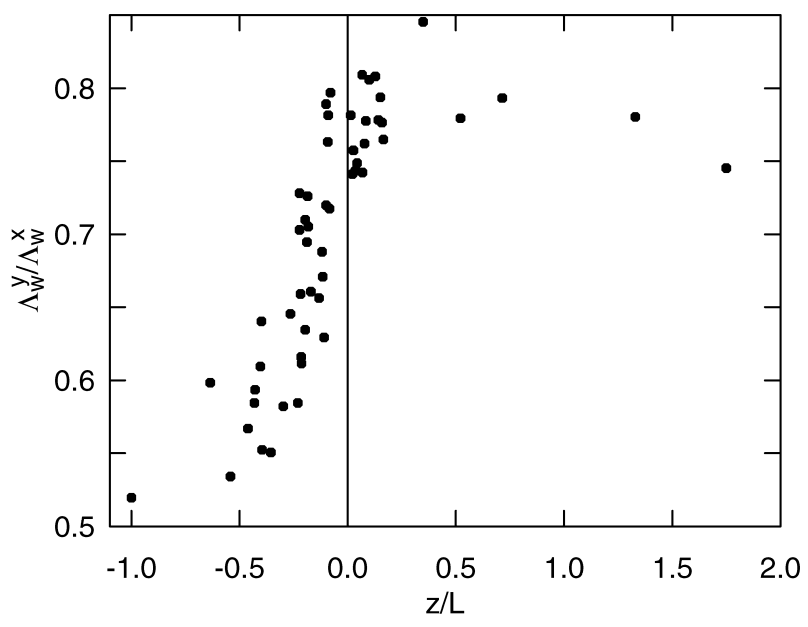

FIG. A2. The ratio of the wavelengths $\Lambda_{w}$ at the peaks of the crosswind and streamwise spectra of vertical velocity, as a function of $z / L$.

\section{APPENDIX B}

\section{LES Model Description}

The LES is based on the numerical approach described in Albertson and Parlange (1999, 2000), which uses pseudospectral treatment in horizontal planes (periodic boundary conditions) and second-order finite differencing in the vertical direction. A neutral atmospheric boundary layer is simulated on an $N^{3}=120^{3}$ grid, which is staggered in the vertical direction. In the output, the vertical velocity is linearly interpolated from the staggered grid nodes for vertical velocities to the horizontal velocity nodes. Nonlinear advection terms and SFS stress are dealiased using the $3 / 2$ rule. The flow is driven by a constant pressure gradient in the streamwise direction, implying a prescribed friction velocity $u_{*}$. The domain size is $2 \pi H \times 2 \pi H \times H$, with horizontal meshspacing $\delta=2 \pi H / N$. The top boundary at $z=H$ is specified to be stress free. At the ground, a traditional log-layer boundary condition is used in which the wall shear stress is prescribed (Moeng 1984). The surface roughness parameter is chosen to be $z_{0} / H=10^{-4}$. The mean velocity at the top of the simulation domain is $\left\langle u_{1}(z=H)\right\rangle / u_{*}=21.94$.

Turbulence at scales smaller than $\delta$ is parameterized with the scale-dependent dynamic model (Porté-Agel et al. 2000a), which determines the Smagorinsky coefficient $c_{s}$ self-consistently from the resolved scales in the simulation. The model adjusts to local conditions without assuming that the coefficient is scale invariant or prescribing ad hoc wall-damping functions. The computed Smagorinsky coefficient is approximately constant in the more isotropic turbulence region away from the ground $\left(c_{s} \sim 0.13\right.$ for $\left.z / \delta>2\right)$, but decreases sharply with decreasing $z$ near the surface. The dimensionless velocity gradient $\Phi=\left(\kappa z / u_{*}\right) \partial U / \partial z$ remains near unity at the ground, while the energy spectra show the ex- pected $k^{-5 / 3}$ slope for inertial scales. For the analysis of Taylor's hypothesis, $u_{i}(x, y, z, t)$ are stored every $t=$ $1.6 \times 10^{-4} \mathrm{H} / u_{*}$ for a total duration of $0.08 \mathrm{H} / u_{*}$, that is 500 samples over about 0.3 domain-crossing times at the mean velocity at the top of the domain.

\section{REFERENCES}

Albertson, J. D., and M. B. Parlange, 1999: Surface length-scales and shear stress: Implications for land-atmosphere interaction over complex terrain. Water Resour. Res., 35, 2121-2132.

— and - 2000: Natural integration of scalar fluxes from complex terrain. Adv. Water Resour., 23, 239-252.

Fisher, M. J., and P. O. A. L. Davies, 1963: Correlation measurements in a non-frozen pattern of turbulence. J. Fluid Mech., 18, 97116.

Hinze, J. O., 1959: Turbulence: An Introduction to Its Mechanism and Theory. McGraw-Hill, $586 \mathrm{pp}$.

Kaimal, J. C., and J. J. Finnigan, 1994: Atmospheric Boundary Layer Flows. Oxford University Press, $189 \mathrm{pp}$.

—_ J. C. Wyngaard, Y. Izumi, and O. R. Coté, 1972: Spectral characteristics of surface-layer turbulence. Quart. J. Roy. Meteor. Soc., 98, 563-589.

,$- \ldots$, D. A. Haugen, O. R. Coté, Y. Izumi, S. J. Caughey, and C. J. Readings, 1976: Turbulence structure in the convective boundary layer. J. Atmos. Sci., 33, 2152-2169.

— , R. A. Eversole, D. H. Lenschow, B. B. Stankov, P. H. Kahn, and J. A. Businger, 1982: Spectral characteristics of the convective boundary layer over uneven terrain. J. Atmos. Sci., 39, $1098-1114$.

Kleissl, J., C. Meneveau, and M. B. Parlange, 2003: On the magnitude and variability of subgrid-scale eddy-diffusion coefficients in the atmospheric surface layer. J. Atmos. Sci., 60, 2372-2388.

Leonard, A., 1974: Energy cascade in large eddy simulations of turbulent fluid flows. Advances in Geophysics, Vol. 18, Academic Press, 237-248.

Lumley, J. L., 1965: Interpretation of time spectra measured in highintensity shear flows. Phys. Fluids, 8, 1056-1062.

Mason, P. J., 1994: Large-eddy simulation: A critical review of the technique. Quart. J. Roy. Meteor. Soc., 120, 1-26.

Meneveau, C., and J. Katz, 2000: Scale invariance and turbulence models for large-eddy simulations. Annu. Rev. Fluid Mech., 32, $1-32$.

Moeng, C.-H., 1984: A large-eddy-simulation model for the study of planetary boundary-layer turbulence. J. Atmos. Sci., 41, 20522062.

Murray, J. A., U. Piomelli, and J. M. Wallace, 1996: Spatial and temporal filtering of experimental data for a-priori studies of subgrid-scale stresses. Phys. Fluids, 8, 1978-1980.

Nicholls, S., and C. J. Readings, 1981: Spectral characteristics of surface layer turbulence over the sea. Quart. J. Roy. Meteor. Soc., 107, 591-614.

Nieuwstadt, F. T. M, P. J. Mason, C.-H. Moeng, and U. Schumann, 1993: Large-eddy simulation of the convective boundary layer: A comparison of four computer codes. Turbulent Shear Flows, Dursts et al., Eds., Vol. 8, Springer-Verlag, 343-367.

Panofsky, H., and J. Dutton, 1984: Atmospheric Turbulence: Models and Methods for Engineering Applications. Wiley, $397 \mathrm{pp}$.

Pope, S. B., 2000: Turbulent Flows. Cambridge University Press, 771 pp.

Porté-Agel, F., C. Meneveau, and M. B. Parlange, 2000a: A scaledependent dynamic model for large-eddy simulation: Application to the atmospheric boundary layer. J. Fluid Mech., 415, 261-284

_ M. B. Parlange, C. Meneveau, W. E. Eichinger, and M. Pahlow, 2000b: Subgrid-scale dissipation in the atmospheric surface layer: Effects of stability and filter dimension. J. Hydrometeor., 1, $75-87$. , and _ 2001: A priori field study of the subgrid- 
scale heat fluxes and dissipation in the atmospheric surface layer J. Atmos. Sci., 58, 2673-2698.

Powell, D. C., and C. E. Elderkin, 1974: An investigation of the application of Taylor's hypothesis to atmospheric boundary layer turbulence. J. Atmos. Sci., 31, 990-1002.

Sullivan, P. P., T. W. Horst, D. H. Lenschow, C.-H. Moeng, and J. C. Weil, 2003: Structure of subfilter-scale fluxes in the atmospheric surface layer with application to LES modeling. J. Fluid Mech., 482, 101-139.

Taylor, G. I., 1938: The spectrum of turbulence. Proc. Roy. Soc. London, A164, 476-490.
Tong, C., J. C. Wyngaard, S. Khanna, and J. G. Brasseur, 1998: Resolvable- and subgrid-scale measurement in the atmospheric surface layer: Technique and issues. J. Atmos. Sci., 55, 31143126.

—,- , and J. G. Brasseur, 1999: Experimental study of the subgrid-scale stresses in the atmospheric surface layer. J. Atmos. Sci. 56, 2277-2292.

Willis, G. E., and J. W. Deardorff, 1976: On the use of Taylor's translation hypothesis for diffusion in the mixed layer. Quart. J. Roy. Meteor. Soc., 102, 817-822.

Wyngaard, J. C., and S. F. Clifford, 1977: Taylor's hypothesis and high-frequency turbulence spectra. J. Atmos. Sci., 34, 922-929. 